\title{
Enhanced oral bioavailability and hepatoprotective activity of thymoquinone in the form of phospholipidic nano-constructs
}

\begin{abstract}
Background: The poor biopharmaceutical properties of thymoquinone (TQ) obstruct its development as a hepatoprotective agent. To surmount the delivery challenges of TQ, phospholipid nanoconstructs (PNCs) were constructed.
\end{abstract}

Method: PNCs were constructed employing microemulsification technique and systematic optimization by three-factor three level Box-Behnken design.

Result: Optimized PNC composition exhibited nano size $(<100 \mathrm{~nm})$, spherical morphology, within acceptable range of polydispersity index (0.55), high drug entrapment efficiency (>90\%), controlled drug release pattern, and neutral surface charge (zeta potential of $-0.65 \mathrm{mV}$ ). After oral administration of a single dose of PNC, it showed a relative bioavailability of $386.03 \%$ vis-à-vis plain TQ suspension. Further, TQ-loaded PNC demonstrated significant enhanced hepatoprotective effect vis-à-vis pure TQ suspension and silymarin, as evidenced by reduction in the ALP, ALT, AST, bilirubin and albumin level and ratified by histopathological analysis.

Conclusion: TQ-loaded PNCs can be efficient nano-platforms for the management of hepatic disorders and promising drug delivery systems to enhance oral bioavailability of this hydrophobic molecule

KEYWORDS: Thymoquinone; Box-Behnken design; lipid carriers; microemulsifcation; hepatotoxicity; pharmacokinetics 


\section{Graphical abstract}
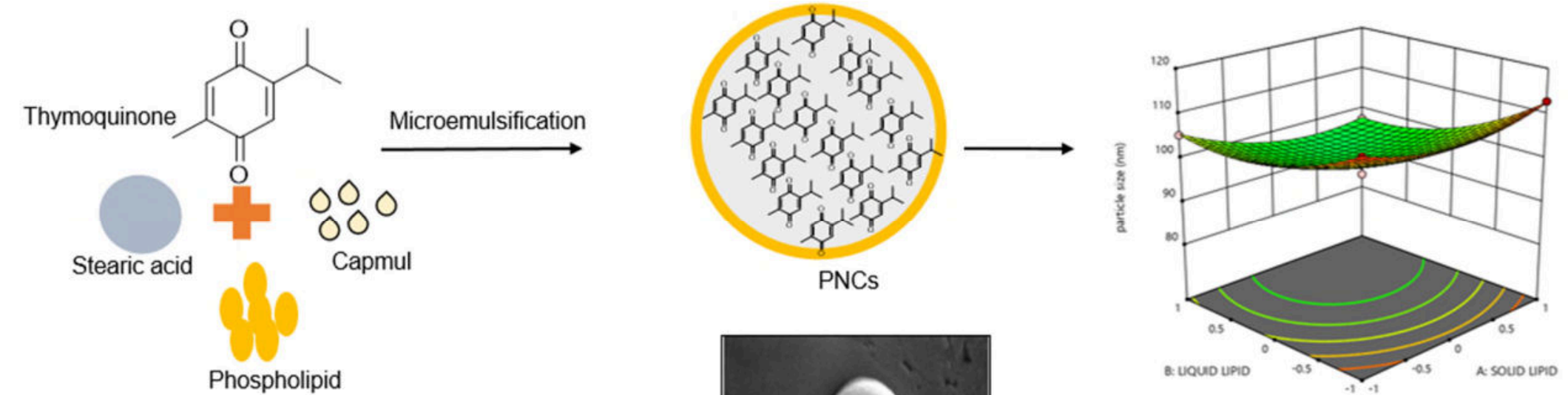

Phospholipid
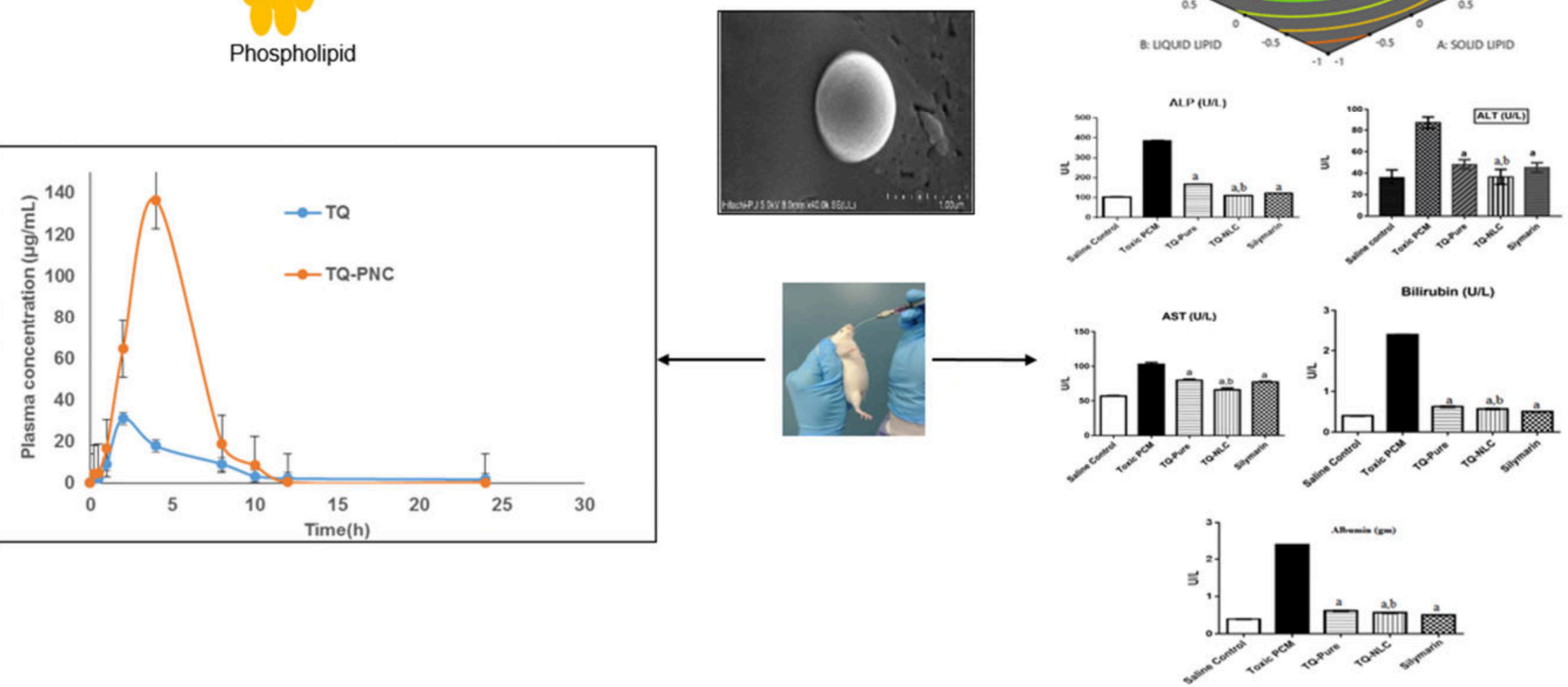


\section{Introduction}

Liver diseases, especially viral hepatitis occurs predominately in developing countries with an enormous impact on public health and economy [1,2]. Liver diseases account for approximately 2 million deaths per year worldwide [3]. Thymoquinone (TQ: 2-isopropyl-5-methylbenzo-1,4quinone), the main active molecule of Nigella sativa (NS) essential oil, is a promising contender for the prevention of hepatic diseases [4-6]. The hepatoprotective effects of TQ may be linked with its ameliorating effects on inflammation, apoptosis, and oxidative stress pathways. This subsequently results in the less transactivation of hepatic stellate cells (HSCs) [2,7,8]. Oral administration of TQ alleviates oxidative stress in liver mucosa directly via its free radical scavenging action and/or indirectly via stimulation of endogenous enzymatic and non-enzymatic antioxidants enzymes [9]. Further, TQ alleviates liver inflammation by inhibiting cyclooxygenase pathway and eicosanoid generation in leukocytes, and also 5-lipooxygenase and peroxidase [10]. Although, TQ is a potential candidate for treating hepatic diseases but its highly hydrophobic nature limits its oral bioavailability. The solubility of TQ in aqueous medium has been reported to be less than $<1.0 \mathrm{mg} / \mathrm{mL}$ at room temperature. Further, it is also reported to have degradation in the aqueous media and in the presence of light. These limitations can be overcome by strategically formulating nanosized formulation of TQ such as phospholipid nanoconstructs, which can protect the encapsulated drug, enhance the bioavailability, passively-target the drug to the liver, and release the drug in a controlled and effective manner.

Phospholipid based nanocarrier systems could be a potential delivery system for enhancing solubility and therapeutic efficacy (11). Presently, researchers are being focused to the development of newer delivery approaches to get enhanced benefit using TQ.

Kalam et al., 2017 evaluated the hepato-protective potential of TQ-loaded self-nanoemulsifying drug delivery systems (SNEDDS) using $\mathrm{CCl}_{4}$ induced hepato-toxicity model. Results indicated the significant hepatoprotective effects for optimized TQ-loaded SNEDDS vis-à-vis TQ suspension. The comparative bioavailability of TQ was enhanced 3.87-fold by optimized SNEDDS in comparison with TQ suspension. (Singh et al., 2013) evaluated the hepato-protective potential of TQ-loaded solid lipid nanoparticles (SLNs) against Paracetamol-induced hepatotoxicity. Findings revealed that TQ-SLNs significantly reduced the serum biomarker enzymes (SGOT, SGPT and ALP) compared to pure TQ suspension. Further, in vivo studies revealed a nearly 5-fold increase in the bioavailability of TQ-SLN as compared to pure TQ suspension. 
Among various lipidic nanocarrier systems, nanostructured lipid carriers (NLCs) composed of lipids, blend of surfactant/co-surfactant and water seen to be effective in accomplishing the therapeutic requirements of TQ. Sayeed et al., prepared TQ-loaded proniosome based formulation for hepato-protective activity against methotrexate-induced hepato-toxicity. Findings revealed that TQ-loaded proniosomes significantly inhibited the elevated levels of liver enzymes, serum marker enzymes and improved histopathological abnormalities [12].

NLCs, the second-generation of lipid carriers, are composed of a mixture of spatially different lipids, i.e., the blend of solid lipid and liquid lipid [13,14]. NLCs stimulate oral absorption of encapsulated drugs via selective uptake through the lymphatic route or the payer's patches [1517]. NLC formulations of TQ prepared by high shear homogenization and ultrasonication have earlier been reported to improve the therapeutic efficacy of the drug in animal models for the treatment of gastric ulcer [18] tumor [19] and hepatic disorders [9]. In comparison to the previously employed methods to prepare TQ-loaded NLCs, microemulsification method is simple, reproducible, no energy requirement, cost-effective and can be easily scaled-up. Moreover, NLCs with low particle size, narrow size distribution and high entrapment efficiency can be obtained. The combination of surfactants usually produces smaller particle sizes due to the surface-active property of the surfactants. Thus, in the present research work, we have attempted to incorporate phospholipid (i.e., Phospholipon $90 \mathrm{G}$ ) as surfactant-co-surfactant mixture ( $\left.\mathrm{S}_{\text {mix }}\right)$ with a non-ionic surfactant (i.e., Tween 80) so as to result in the construction of the phospholipid nanoconstructs (PNCs) employing microemulsification method. No report is traceable in the literature regarding the phospholipid based NLCs for TQ. Phospholipon 90G is a Zwitterionic surfactant and is expected to enhance the biopharmaceutical performance and therapeutic efficacy of hydrophobic drug molecules. It tends to provide enhanced solubility and permeability of the drugs across the biological barriers, thus improving their oral biopharmaceutical performance owing to its amphiphilic nature. Additionally, phospholipids constitute a major part of the bio-membrane and thus enhance the cellular uptake of the drugs [20]. Besides this, being amphiphilic compound phospholipids when placed in water from micelles like structure or are organized as lipid bilayers with the hydrophobic tails facing against one another and the hydrophilic head-group facing the water on both sides. These unique features make phospholipids most appropriate to be used as cosurfactant for poorly water-soluble drugs like TQ. Thus, kept in mind that the enhanced oral 
absorption of hydrophobic drugs via intrinsic lipid pathways can be achieved by incorporating the drug into phospholipid based systems.

The best understanding of the product and process during the development of a formulation could be achieved employing design of experiments (DoE), which is an excellent tool to systematically manipulate factors according to a prespecified design [21]. Box-Behnken design (BBD), is one such DoE response surface methodology design, having numerous design advantages in comparison to other second order designs, e.g., central composite design (CCD) [22-24]. Thus, in the present investigation, BBD was used to optimize the TQ-loaded PNCs. Solid lipid, liquid lipid and surfactant/co-surfactant blend i.e., $\left(\mathrm{S}_{\mathrm{mix}}\right)$ : (T80:PL90G), were employed as the input variables for studying their effect on the responses like particle size and entrapment efficiency. The bestoptimized TQ-loaded PNC was then evaluated for in vitro release kinetics to study the release mechanism, pharmacokinetic assessment and hepato-protective activity in Paracetamol-induced hepato-toxicity model in Wistar rats.

\section{Materials and Methods}

\subsection{Materials}

TQ was purchased from M/s Sigma Aldrich, Mumbai, India. Phospholipon 90G (PL90G) was received ex gratis from M/s Phospholipid GmbH, Nattermannallee, Germany. Ethanol was purchased from M/s Merck Specialities Pvt. Ltd., Mumbai, India, while Span 80 and Tween 80 from M/s Qualikem Fine Chemicals Pvt. Ltd. Vadodara, India, Glyceryl monostearate (GMS) and stearic acid were obtained from Hi-Media Pvt. Ltd., Mumbai, India, and Capmul was procured from, M/s Biochem Pharmaceutical Industries. Olive oil, castor oil and oleic acid were purchased from M/s Loba Chemie Pvt. Ltd., Mumbai, India.

Wistar rats (250-300 g) used in the current study were procured from Animal house facility of National Institute of Pharmaceutical Education and Research, Mohali, Punjab, India and kept at the animal house establishment of Shoolini University, Solan, HP, India. Animal studies were conducted with prior approval of Institutional Animal Ethical Committee of Shoolini University (IAEC/SU/17/07). Rats were housed in plastic bottom cages and allowed free access to standard animal feed and water. The animals were maintained at a temperature of $25 \pm 2{ }^{\circ} \mathrm{C}$ with relative 
humidity of $45 \pm 5 \%$. The animals were initially acclimatized to experimental laboratory conditions for 7 days before the experiments.

\subsection{Screening of liquid lipids}

Selection of various liquid lipids viz. olive oil, capmul, oleic acid, and castor oil was done based on their solubility in TQ. Direct exposure of the drug to sunlight was avoided by working carefully in a dark hood within the laboratory. About $10 \mathrm{~mL}$ of oil was placed in $15 \mathrm{~mL}$ culture tubes and excess amount of TQ was added into it maintained at thermostatically controlled shaker water bath maintained at $37 \pm 1^{\circ} \mathrm{C}$. Further, the weighed quantity of clear solution was dissolved in ethanol and analyzed spectrophotometrically after suitable dilution (s).

\subsection{Screening of solid lipids}

Solubility studies for TQ were carried out in various lipids, viz. Compritol, stearic acid and GMS. Excess amount of drug was added into $100 \mathrm{mg}$ of solid lipid taken in a $5 \mathrm{~mL}$ culture tube maintained at thermostatically controlled shaker water bath at $70 \pm 1^{\circ} \mathrm{C}$ to get a clear solution. Further, the weighed quantity of clear solution was dissolved in ethanol and analyzed spectrophotometrically after suitable dilution (s). Whole study was carried in a dark hood within the laboratory to protect the drug from direct exposure to sunlight.

\subsection{Preparation of PNCs}

PNCs were constructed employing microemulsifcation technique with some modification. Initially, drug was dissolved in a portion of ethanol. The phospholipid was dispersed/dissolved in a portion of water along with Tween 80 to form the aqueous phase. Solid lipid was melted at $70^{\circ} \mathrm{C}$ and liquid lipid was completely mixed with it. Subsequently, lipid phase, aqueous phase and drug solution were mixed isothermally to obtain a clear microemulsion. The remaining portion of water was cooled at $4^{\circ} \mathrm{C}$. The hot microemulsion so formed was poured into ice-cold water previously maintained at $4{ }^{\circ} \mathrm{C}$ and stirred continuously for $20 \mathrm{~min}$ at $3000 \mathrm{rpm}$ to get the PNCs [15, 25-28] .

\subsection{Systematic optimization and validation of PNCs}

TQ-loaded PNCs were optimized using three-level, Box-Behnken design (BBD). The selected dependent variables were the amount of solid lipid $\left(\mathrm{X}_{1}\right)$, liquid lipid $\left(\mathrm{X}_{2}\right)$, and $\mathrm{S}_{\text {mix }}\left(\mathrm{X}_{3}\right)$, employed at three different levels of each variable, viz. low (-1), intermediate (0), and high (+1). Various PNC formulations (Table 1) prepared as per the design were investigated for the response variables 
viz. particle size, and percent entrapment efficiency (\%EE). Optimization data analysis was performed after evaluating prepared PNCs for the response variables. The response surface analysis was studied employing three dimensional (3D) response surface plots, and twodimensional (2D) contour plots, generated using Design Expert ${ }^{\circledR}$ ver.10.0.1 (MS Stat-Ease Inc., Minnepolis, MN) [24,28].

Validation of methodology was done by selecting 17 PNCs formulation from the numerical optimisation technique based on desirability function. The validated formulations were evaluated, and the observed and predicted values were critically compared for the responses.

\subsection{Particle size determination and zeta potential}

The particle size of the prepared PNC's was determined by dynamic light scattering (Zetasizer 2000 HS, Malvern Instruments Limited, Malvern, UK), installed at Chemistry Department, Panjab University, India. Whereas, zeta potential was measured by Delsa Nano C (Beckman Coulter LS13320), installed at University Institute of Pharmaceutical Sciences (UIPS) India. The formulations $(0.1 \mathrm{~mL})$ were diluted up to $100 \mathrm{~mL}$ with distilled water and mixed well before analysis at room temperature. The measurements were made at a detection angle of $90^{\circ}$, and the measurement position within the cuvette was automatically determined by the software. Approximately $1 \mathrm{~mL}$ of the sample was placed in the cuvette and the intensity of fluctuation of the laser beam was recorded, correlated with the particle size of the dispersed phase [29].

\subsection{Percent Entrapment efficiency (\%EE)}

The \%EE of TQ-PNC was determined by measuring free TQ in the aqueous phase, which was separated using ultrafiltration centrifugation. Approximately $1 \mathrm{~mL}$ of TQ-PNCs was centrifuged at $3000 \mathrm{rpm}$ for $20 \mathrm{~min}$. and filtered using Filter Unit $100 \mathrm{kDa}$ (Millipore Co., Boston, USA). The supernatant $(0.1 \mathrm{~mL})$ was dissolved in ethanol $(1 \mathrm{~mL})$, and analyzed using HPLC method. \%EE of TQ was calculated using Eq. (1)

$$
\% E E=\frac{(T-C)}{T} X 100
$$

Wherein $\mathrm{T}$ is the total amount of drug detected in both the supernatant and sediment, and $\mathrm{C}$ is the amount of drug detected only in the supernatant 


\subsection{Diffraction scanning calorimetry (DSC)}

The DSC pattern of pure TQ, freeze dried TQ-loaded PNC, sucrose, Capmul and stearic acid were recorded via. NETZSCH leading Thermal Analysis (DSC 204, F1 Phoenix ASC) (Germany). The samples (drug, lipid and formulation) were scanned in an aluminium pan at heating rate of 10 ${ }^{\circ} \mathrm{C} / \mathrm{min}$ over the temperature range of $20-350{ }^{\circ} \mathrm{C}$ under an inert argon atmosphere at a flow rate of $20 \mathrm{~mL} / \mathrm{min}$. to evaluate the stability and compatibility

\subsection{Electron Microscopy}

Transmission electron microscopy was used to determine the morphology of optimized PNC with the help of transmission electron microscope (TEM) installed at Indian Institute of Technology (IIT), Mandi, H.P., India. The PNCs were negatively stained with 1\% (w/w) aqueous solution of phosphotungstic acid, and dried on a microscopic carbon-coated grid, and viewed under the microscope at suitable magnifications.

Field emission-scanning electron microscopy (FE-SEM) of the lyophilized sample was also performed to see the morphology of TQ-loaded PNCs. Samples were located on carbon conductive tap and placed inside the vacuum chamber [30]. Moving electron from tungsten filament was made the incident to the sample and surface morphology of nanoconstructs was determined by using FESEM (Hitachi s-4800), installed at SAIF, Panjab University, Chandigarh, India.

\section{8. $X$-ray diffraction $(X R D)$}

The XRD pattern of pure TQ, freeze dried TQ-loaded PNC, sucrose, Capmul and stearic acid were recorded via. X'Pert PRO diffractometer system (Panalytical, Netherlands) with a Copper Ka radiation $(1.54060$ A) [31]. The tube voltage and current were fixed at $45 \mathrm{kV}$ and $40 \mathrm{~mA}$ respectively. Each samples was placed in an aluminum container and measured by a continuous scan between 5 and $40^{\circ}$ in $2 \theta$ with a step size of 0.017 [2].

\subsection{FTIR spectroscopy}

Infrared spectroscopy was performed by Attenuated total reflection Fourier transform infrared spectrometer (ATR-FTIR) to determine interaction between excipients, and the active drug i.e., TQ. 


\subsection{In vitro drug release kinetics}

In vitro drug release was performed via dialysis membrane method. The membrane was made into pouches by tying the thread on both the ends, and both pure TQ suspension and equivalent TQloaded PNCs were placed into the bags separately. The dialysis bags were then placed in a beaker containing in $25 \mathrm{~mL}$ of release media kept in water bath shaker maintained at $37^{\circ} \mathrm{C}$ and run at 100 strokes/min [32]. For first $2 \mathrm{~h}$, release was carried out in $0.1 \mathrm{~N} \mathrm{HCl}(\mathrm{pH} 1.2)$ followed by phosphate buffer (pH 6.8) up to $12 \mathrm{~h}$ along with $2.5 \%$ w/v of Tween 80 . Aliquots of $2 \mathrm{~mL}$ were withdrawn at regular time intervals and replaced with an equal amount of diffusion medium to maintain the sink conditions. Samples were then analyzed using HPLC at $\lambda_{\max }$ of $254 \mathrm{~nm}$ after appropriate dilution(s).

To study the release mechanism of the drug from PNC, the in vitro release data was fitted into various mathematical models viz. zero order, first order, Higuchi model and Korsermeyer Peppas model [33]. The kinetic model was selected on the best fit with the highest value of regression coefficient $\left(\mathrm{r}^{2}\right)$.

2.11. In vitro drug release kinetics in simulated gastric fluid (SGF) and simulated intestinal fluid $(S I F)$

In vitro drug release was performed via dialysis membrane method. The membrane was made into pouches by tying the thread on both the ends, and both pure TQ suspension and equivalent TQloaded PNCs were placed into the bags separately. The dialysis bags were then placed in a beaker containing in $25 \mathrm{~mL}$ of release media kept in water bath shaker maintained at $37^{\circ} \mathrm{C}$ and run at 100 strokes/min [32]. For first 2 h, release was carried out in SGF (pH 1.2) followed by SIF (pH 6.8) up to $8 \mathrm{~h}$ along with $2.5 \% \mathrm{w} / \mathrm{v}$ of Tween 80 . Aliquots of $1 \mathrm{~mL}$ were withdrawn at regular time intervals and replaced with an equal amount of fresh diffusion medium to maintain the sink conditions. Samples were then analyzed using HPLC at $\lambda_{\max }$ of $254 \mathrm{~nm}$ after appropriate dilution(s) and the cumulative amount of drugs released was calculated.

\subsection{Stability studies}

The stability of the developed formulation was studied in simulated gastrointestinal (GI) fluids (SGF pH 1.2 and SIF pH 6.8). Briefly, 10ml of simulated GI fluids were added into $2 \mathrm{~mL}$ of PNC and incubated for $2 \mathrm{~h}$ in case of SGF (pH 1.2) and for $6 \mathrm{~h}$ in case of SIF (pH 6.8). Following the incubation, the formulation was evaluated for particle size, PDI and \%EE. 


\subsection{Caco-2 cell culture studies}

\subsubsection{Qualitative Uptake study}

For the qualitative cell uptake study the Caco-2 cells were employed at a density of 50,000 cells/well in 6 well plate (Costars, corning Inc., NY, USA). The harvested cells were seeded and kept overnight for the attachment of cells. Afterward, the incubation of the cells was done with free Coumarin 6 (C-6) and C-6 loaded PNC (equivalent to $1 \mu \mathrm{g} / \mathrm{mL}$ of free C-6) for $3 \mathrm{~h}$. The medium was removed after the incubation was finished. Further, Hank's balanced salt solution (HBSS) was used for washing the cells thrice followed by observing under the Confocal Laser Scanning Microscope (CLSM) (Olympus FV 1000) [34].

\subsubsection{Quantitative uptake}

The quantitative cell uptake study was performed as per the previous report [34]. Briefly, Caco-2 cells were seeded at a density of 1x105 cells/well in six well cell culture plates (Costars, Corning Inc., NY USA). The medium of cell culture was replaced with fresh medium having a set concentration $(10 \mu \mathrm{g} / \mathrm{mL})$ of native TQ and PNC and further incubated for variable time intervals of $0.5 \mathrm{~h}$ to $3 \mathrm{~h}$ to evaluate the time dependent cell uptake. After, the period of incubation was accomplished the pre-existing medium was discarded followed by washing of cells thrice by HBSS. Further, the lysis of the cells was done with $0.1 \%$ Triton $^{\mathrm{TM}} \mathrm{X}-100$ and methanol was employed for extraction in order to solubilize the drug, which was internalized. Finally, the centrifugation (Sigma K 300, USA) of cell lysates, which were obtained, was done at 34,000 rcf for a period of $10 \mathrm{~min}$. The supernatant obtained was then quantified for TQ with the aid of validated HPLC method.

\subsection{In vivo studies}

\subsubsection{Oral pharmacokinetics}

The rats were randomly divided into two groups $(n=6)$. The first group was orally administered with TQ $(20 \mathrm{mg} / \mathrm{kg})$ dispersed in $4 \%$ sodium carboxymethylcellulose $(\mathrm{CMC}-\mathrm{Na})$ and for second group single dose of TQ-loaded PNCs $(20 \mathrm{mg} / \mathrm{kg})$ was administered. Blood samples of about 0.5 $\mathrm{mL}$ each were withdrawn from the retro-orbital plexus under light anaesthesia at specified time intervals (pre-dose, $0.5,1,2,4,6,8,10,12,18$, and $24 \mathrm{~h}$ ), placed into heparinized eppendorff 
tubes and instantly centrifuged at $3000 \mathrm{rpm}$ for $15 \mathrm{~min}$. [2, 26]. After centrifugation, the plasma obtained was stored at $-40{ }^{\circ} \mathrm{C}$ until further analysis. Extraction of TQ from rat plasma was performed and analyzed employing HPLC. The plasma obtained was stored at $-40{ }^{\circ} \mathrm{C}$ until further analysis. An aliquot of $200 \mu \mathrm{L}$ of plasma-containing drug (TQ) was then placed in centrifuge tube and precipitated with equal volume of acetonitrile $(200 \mu \mathrm{L})$. Further, methanol was added to make up the volume up to $1 \mathrm{~mL}$. The mixture was then centrifuged at $6000 \mathrm{rpm}$ for about $10 \mathrm{mins}$. Supernatant $(20 \mu \mathrm{L})$ was injected onto the column for the analysis of TQ employing HPLC [35]. The pharmacokinetic analysis was performed by fitting the obtained data into one compartment open body model (1CBM) extravascular model and various pharmacokinetic parameters Cmax, $\mathrm{t}^{1 / 2}, \operatorname{tmax}, \mathrm{V}_{\mathrm{d}}, \operatorname{AUC}(0-24), \operatorname{AUC}(0-\infty)$ and $\mathrm{F}$ were calculated using Microsoft Excel add-in, "PKSolver".

\subsection{PCM-induced hepato-toxicity}

The rats were randomly divided into 5 groups $(\mathrm{n}=4)$ namely normal control group (Group I), which were provided a daily dose of $1 \mathrm{~mL}$ aqueous solution of $0.9 \%$ w/v sodium chloride $(\mathrm{NaCl})$ (p.o.) for 7 days. Toxic group (Group II) received $(650 \mathrm{mg} / \mathrm{kg}$ ) PCM once daily for 7 days to induce hepatic damage. TQ-suspension (Group III) dispersed in $4 \%$ sodium carboxymethylcellulose (CMC-Na) $(1 \mathrm{~mL})(20 \mathrm{mg} / \mathrm{kg})$ were administered orally, TQ-loaded PNC suspension group (Group IV) received (20mg/kg) daily dose for 7 days, and standard group treated with an oral suspension of Silymarin tablet (SILYBON®) $(40 \mathrm{mg} / \mathrm{kg}$ ) for 7 days (Group V) $(40 \mathrm{mg} / \mathrm{kg}$ ). The rats in the toxic group (Group II) was given oral dose of $650 \mathrm{mg} / \mathrm{kg}$ (PCM) for seven days. All the groups, including toxic group (group II), except group I (control) received concurrent dose of $650 \mathrm{mg} / \mathrm{kg}$ PCM p.o for 7 days, while the rats in the normal control group was given the vehicle (normal saline) only. After 7 days, about $1 \mathrm{~mL}$ of the blood sample was withdrawn from the retro orbital plexus under light ether anaesthesia. The blood was collected in non-heparinized tubes, centrifuged at $1500 \mathrm{rpm}$ for $15 \mathrm{~min}$, the serum was separated and frozen at $-80^{\circ} \mathrm{C}$ until further testing. The serum so obtained was analyzed for various enzymes such as alanine aminotransferase (ALT), aspartate aminotransferase (AST), alkaline phosphate (ALP), bilirubin and albumin respectively [12,36-37]. 


\subsection{Histopathological examination}

Liver tissue samples were excised on the last day of the study. The samples were washed with phosphate buffer saline, dried with tissue paper and preserved in $10 \%$ formalin solution. These liver sections were stained with haematoxylin and eosin (H\&E) and examined under a light microscope for the presence of any necrosis, haemorrhage, and inflammatory cells.

\section{Statistical analysis}

The data obtained are reported as mean \pm SD. Data statistical analysis was performed by Graph Pad Prism 8.0. The data from different formulations were compared for statistical significance by the one-way analysis of variance (ANOVA) followed by Tukey's multiple comparison Test. A difference was considered statistically significant at the level of $p<0.05$.

\section{Results}

\subsection{Formulation and optimization PNC formulation}

The maximum solubility of TQ was obtained in the stearic acid (solid lipid) and capmul (liquid lipid) (data not shown), thus selected for the development of PNCs. For the optimization of TQloaded PNCs, a three-factor, three-level Box-Behnken design was employed [24]. Table 1 summarises an account of the 17 experimental runs studied, along with the coded values and actual values for the studied factors. The \%EE, and particle size values obtained for the various PNC formulations are enumerated in table 1 and figure 1 (a-f)

The data analysis was carried out by analysing the response variables employing second-order quadratic polynomial models $(\mathrm{p}<0.0001)$ [28]. The $\mathrm{r}^{2}$ values for these models (particle size $=$ 0.9903; \% $\mathrm{EE}=0.9909$ ) indicating excellent goodness of fit. The models was also found to have insignificant values of "lack of fit" endorsing the relevance of proposed model was appropriate. Closeness in the magnitude of adjusted and predicted $r^{2}$ to the actual model $r^{2}$ also supported an excellent good of fit to the data.

\subsubsection{Model Generation}

The data analysis was carried out by analysing the response variables employing second-order quadratic polynomial models $(\mathrm{p}<0.0001)$ [26]. Application of ANOVA suggested that the chosen quadratic model was highly significant $(\mathrm{p}<0.05)$ along with the model terms $(\mathrm{p}<0.0001)$. The $\mathrm{r}^{2}$ 
values for these models (particle size $=0.9903 ; \% \mathrm{EE}=0.9909$ ) indicating excellent goodness of fit. The models was also found to have insignificant values of "lack of fit" endorsing the relevance of proposed model was appropriate. Closeness in the magnitude of adjusted and predicted $\mathrm{r}^{2}$ to the actual model $\mathrm{r}^{2}$ also supported an excellent good of fit to the data. The special polynomial mathematical model encompassing ten coefficients $(\beta 0-\beta 33)$ was postulated with $\beta 0$ as the intercept. All the coefficients represent quadratic and interaction terms, as depicted in Eq. 2

$Y=\beta_{0}+\beta_{1} X_{1}+\beta_{2} X_{2}+\beta_{3} X_{3}+\beta_{12} X_{1} X_{2}+\beta_{13} X_{1} X_{3}+\beta_{23} X_{2} X_{3}+\beta_{11} X_{1}^{2}+\beta_{22} X_{2}^{2}+\beta_{33} X_{3}^{2}$

\subsubsection{Model Optimization}

Optimization was done based on desirability function in order to get the desired goal for the response variables. The optimum formulation was selected based on the criteria, i.e., minimum value of particle size and maximum value of \%EE by applying constraints. The desirability function, ' $r$ ' near or equal to unity was assigned as an indicator of the best-fit composition with desired features. Table 2 presents various constraints and possible solutions with their numeric range of desirability.

\subsubsection{Model Validation}

A total of 7 check-point formulations were chosen from the RSM, evaluated and the observed data compared with the predicted responses (Table 3). Linear correlation plots drawn between the actual and the predicted response variables demonstrated high values of $\mathrm{r}^{2}$ (particle size $=0.9879$; $\% \mathrm{EE}=0.9552)$ indicating excellent goodness of fit. The overall bias for response variables found to be $-0.044 \pm 0.096 \%$, indicating very high degree of predictive ability of the QbD experiments. The residual plots were found to be quite regulated, as they showed uniform, relatively narrow and random scatter around the zero-axis.

\subsection{Characterization of optimized PNCs}

Particle size of the optimized PNC was less than 100nm (i.e. $83.5 \mathrm{~nm}$ and \%EE of the optimized PNC was found to be $94.4 \%$. The particle size distribution (PDI) was found to be 0.55 , which is in acceptable range [38], as shown in Figure 2(a). The zeta potential value was obtained to be -0.64 . 
DSC analysis revealed the melting point of TQ, stearic acid, capmul and PNC. DSC thermogram of TQ showed sharp endothermic peak around $47^{\circ} \mathrm{C}$, which corresponds to its melting point range, as mentioned in the literature $\left(45-50{ }^{\circ} \mathrm{C}\right)$. Whereas, stearic acid, capmul and PNC exhibits a sharp endothermal peak at $60{ }^{\circ} \mathrm{C}, 150{ }^{\circ} \mathrm{C}$ and $165^{\circ} \mathrm{C}$ respectively as shown in figure 2 (b). Whereas (figure 2(c) and (d) shows the TEM and FE-SEM photomicrographs of the developed nanocarrier system, respectively. The TEM images of optimized PNCs reveal that the particles were spherical in shape, in agreement with the size obtained using DLS i.e. below $100 \mathrm{~nm}$. The SEM images also displayed nearly spherical topography of prepared PNC's. No crystalline drug particles were visible as shown in Figure 2(c,d). The XRD pattern of pure TQ, TQ-PNC, sucrose, Capmul and stearic acid is displayed in Figure 2(e). The XRD pattern of the pure TQ demonstrates high intensely peak, showing its crystalline nature, while stearic acid and Capmul exhibited comparatively low intensity peaks indicating their amorphous nature. TQ-PNCs demonstrated a marked decrease in the intensity of TQ peak.

For the estimation of any possible chemical interaction among TQ and excipient, FTIR spectra of TQ, Physical mixture, sucrose and stearic acid, Overlay spectra, of drug, the excipients, and physical mixture is represented in Figure 2(f), while their interpretations, are included in the Table 4. The FTIR spectrum of the pure TQ showed the sharp peak at $2970 \mathrm{~cm}^{-1}$ corresponds to (free $\mathrm{CH}$ stretching, $\left.2800-3200 \mathrm{~cm}^{-1}\right)$, the peak present at $1644 \mathrm{~cm}^{-1}$ corresponds to $(\mathrm{C}=\mathrm{O}$ and $\mathrm{C}=\mathrm{C}$ stretch,1670-1820 $\mathrm{cm}^{-1}$ ), the peak present at 1360 corresponds to (-CH bending, $\left.1350-1480 \mathrm{~cm}^{-1}\right)$. The FTIR spectrum of stearic acid exhibited peaks at $3492 \mathrm{~cm}^{-1}$ corresponds to (-OH stretch, 3200$3600 \mathrm{~cm}^{-1}$ ), the peak presented at $2920 \mathrm{~cm}^{-1}$ corresponds to (-CH stretch, 2850-3000 and peak at 1707 corresponds to $\left(\mathrm{C}=\mathrm{O}\right.$ stretch and $\mathrm{C}=\mathrm{C}$ stretch, $\left.1670-1820 \mathrm{~cm}^{-1}\right)$. The FTIR spectrum of sucrose exhibited a sharp peak at $2927 \mathrm{~cm}^{-1}$ corresponds to (-CH stretch, 2850-3000 $\mathrm{cm}^{-1}$ ). The peak presented at $3667 \mathrm{~cm}^{-1}$ corresponds to (-OH stretch, 3500-3700) peak appeared at $1395 \mathrm{~cm}^{-1}$ corresponds to (-CH stretch, 1350-1480). There was a shifting of the peak of TQ benzene ring and carbonyl, group $(\mathrm{C}=\mathrm{C}, \mathrm{C}=\mathrm{O})$ from $1644 \mathrm{~cm}^{-1}$ to and $1771 \mathrm{~cm}^{-1}$ in the physical mixture.

\subsection{In vitro release kinetic}

The release profile of TQ from plain TQ suspension and optimized PNC formulation is shown in Figure $2(\mathrm{~g})$. It is vivid from the results, that there was much better release of the drug from PNCs vis-à-vis plain TQ suspension. Approximately $80 \%$ of the drug was released from TQ-PNCs up to $12 \mathrm{~h}$, whereas, only $50 \%$ of TQ was released from pure TQ suspension. In initial $2 \mathrm{~h}$, approximately 
$31.5 \%$ of the drug was released from PNC vis-à-vis pure TQ (10.8\%) followed by sustained release at a constant rate.

After fitting the release data into various kinetic models, the best-fit model was found to be Higuchi model, which indicated the controlled release behavior of the drug from PNC matrix.

\subsection{In vitro drug release (SGF) and (SIF)}

The release profile of TQ from plain TQ suspension and optimized PNC formulation is shown in Figure 2(h). Almost $100 \%$ of the pure TQ drug release was achieved within $1 \mathrm{~h}$, which indicated the rapid diffusion of TQ. In case of PNCs initial burst release was seen in the first 30 mins which was nearly $40 \%$ of the total amount of TQ released, followed by a sustained release of the remaining TQ over nearly $8 \mathrm{~h}(91.74 \%)$ [Figure 2(h)].

\subsection{Stability of PNC in simulated GI fluids}

As PNC was designed for oral administration, therefore the main goal behind stability study in simulated GI fluid is to first protect the drug from degradation while passing through harsh environment in GI tract. PNC were able to maintain their integrity upon incubation with simulated GI fluids for specified time. The results showed the insignificant changes in all the formulation parameters viz. particle size, PDI and \%EE (Table 5).

\subsection{Qualitative and quantitative cell uptake}

The uptake of PNC were assessed in Caco-2 cells to predict the possibility of GI membrane associated transport. CLSM images of PNC exhibited higher fluorescence vis-à-vis pure TQ. Figure. 2(i) (I) and (II) depicts CLSM images of pure TQ and respectively. Similarly, quantitative uptake of PNC and pure TQ in Caco-2 cells was performed to further validate the outcomes of CLSM analysis. The time dependent cell uptake of pure TQ and PNC by Caco- 2 cells is depicted in Fig. 2(j). The study showed significantly higher uptake $(p<0.05)$ of PNC vis-a-vis pure TQ. In addition, the cellular uptake was improved when there was an increase in the incubation time from 0.5 to $3 \mathrm{~h}$. After $3 \mathrm{~h}$ of interval, the \% cell uptake was $7.2 \%$ and $41.2 \%$ for pure TQ and PNC respectively at concentration $10 \mu \mathrm{g} / \mathrm{mL}$. 


\subsection{Oral pharmacokinetics}

The plasma concentration-time profile graph of optimized PNC formulation exhibited better enhancement in in vivo absorption of TQ vis-à-vis TQ suspension as shown in figure 3 (A). Various pharmacokinetic parameter for TQ-PNC and TQ suspension is entailed in Table 6 . The $t_{\max }$ value was detected to be $3.96 \mathrm{~h}$ for TQ-PNC and $3.0 \mathrm{~h}$ for pure TQ suspension. Whereas, $\mathrm{t}_{1 / 2}$ was found to be $2.60 \mathrm{~h}$ for TQ-PNC and $2.13 \mathrm{~h}$ for pure TQ suspension. Volume of distribution (Vd) was found to $0.077 \mathrm{~L} / \mathrm{kg}$ for PNC and $0.251 \mathrm{~L} / \mathrm{kg}$ for TQ, clearance (CL) was found to be $0.020 \mathrm{~L} / \mathrm{kg} / \mathrm{h}$ for PNC and $0.081 \mathrm{~L} / \mathrm{kg} / \mathrm{h}$ for TQ. $C_{\max }$ value was found to be $97.06 \mu \mathrm{g} / \mathrm{mL}$ for TQ-PNC and 29.83 $\mu \mathrm{g} / \mathrm{mL}$ for pure TQ suspension. While, $\mathrm{AUC}_{(0-\mathrm{t})}$ and $\mathrm{AUC}_{(0-\infty)}$ of TQ-PNC were found to be 965.614 and $976.29 \mu \mathrm{g} . \mathrm{h} / \mathrm{mL}$, respectively, which were highly significant $(\mathrm{P}<0.05)$ in comparison with $\operatorname{AUC}_{(0-\mathrm{t})}(243.53 \mu \mathrm{g} . \mathrm{h} / \mathrm{mL})$ and $\operatorname{AUC}_{(0-\infty)}(244.29 \mu \mathrm{g} . \mathrm{h} / \mathrm{mL})$ of pure TQ suspension. TQ-PNC showed relative bioavailability of $399.64 \%$ based on mean $\mathrm{AUC}_{(0-\infty)}$ compared to TQ-suspension, indicating the improved bioavailability of the drug in the phospholipidic nanoconstructs.

\subsection{Hepatoprotective activity}

\subsubsection{Serum biochemical estimation}

In vivo hepato-protective effect of TQ-PNCs was studied against PCM-induced hepatotoxicity. PCM administration produced a significant hepatotoxicity. Result of biochemical parameters (ALT, AST, ALP, albumin and total bilirubin) for various groups are given in Table 7 and Figure 3 (B). Severe liver damage in the toxic group can be seen by significant increase in the marker enzymes. Compared to the control group and treatment groups (pure TQ, PNC and silymarin group), PCM administration induced significant increase in ALP, ALT, AST, bilirubin and albumin level production ( $\mathrm{p}<0.001$ ). Treatment for seven days caused the reduction in the ALP, ALT, AST, bilirubin and albumin levels in all the treated groups. Plain TQ suspension revealed almost same levels of biomarker enzymes vis-a-vis standard treatment however, encapsulation of TQ in the PNC significantly reduced the liver biomarker enzymes.

\subsection{Histopathological evaluation}

The results of histopathology of normal, toxic control, TQ suspension, optimized PNC and Silymarin group are presented in Figure 3 (C). Normal group animals showed normal liver with no signs of inflammatory cells and necrosis and showed the usual lobular building. While the 
sections of toxic group showed notable changes viz. parenchymal cell injury, lymphocytes and macrophages infiltration including necrosis and degeneration. However, the animals treated with TQ suspension presented no sign of inflammation and necrosis with minor lymphocytic infiltration. While TQ-PNC treated, animals showed normal lobular architecture in a more distinct manner as compared to TQ suspension, and similar to normal group with few lymphocytes and normal vacuoles. The standard treatment group, i.e., Silymarin, showed almost normal appearance of liver parenchyma, however with few scattered foci with no signs of inflammation and necrosis in hepatocytes.

\section{Discussion}

In the recent past, there has been a tremendous resurgence of research on the bioactive components isolated from the plants, for their prophylactic and therapeutic worth. The strong antioxidant and anti-inflammatory effects, has made TQ a convincing candidate for the treatment of hepatoprotective disorders, as evidenced from the previous literature. However, like other plantbased molecules the delivery of TQ via oral route is difficult, owing to its poor biopharmaceutical properties. The aim of this paper was to encapsulate TQ inside the phospholipidic nanoconstructs, which can protect the drug, enhance the bioavailability, and target the drug to the liver and thereby, releasing the drug in a controlled manner. For the development of PNCs, determination of solubility of the drug in solid lipids, and liquid lipid is a critical aspect. The solubility of the drug in lipids is the most significant, as the ability of the PNCs to maintain the drug in solubilized form is largely influenced by the solubility of drug in the lipidic core of the nanoconstructs. To construct the PNCs, phospholipid was employed as the surfactant. However, it is reported that phospholipids are able to increase the crystallization temperature $(\mathrm{Tc})$ of triglycerides by acting as a template for surface heterogenous nucleation. Phospholipid molecules bound to vesicles, exhibit only a limited mobility and thus, fail to immediately cover the newly created interfaces during recrystallization. Due to the low mobility of the phospholipid molecules, sudden lack of emulsifier on the surface of the particle leads to particle aggregation and increase in the particle size of lipidic nanoconstructs [39]. Thus, the blend of nonionic surfactants are required to regulate and balance the crystallization, which can further stabilize the particle size of nanoconstructs [40].

TQ-loaded PNC formulations were optimized employing BBD to get the best optimal composition, and investigated for \%EE, and particle size as the response variables. In the current studies, all the 
input variables, i.e., the amount of solid lipid, liquid lipid and $S_{\text {mix }}$ had a significant impact on the particle size. A nonlinear dip in the values of particle size followed by a rising trend was observed with increase in the liquid lipid and solid lipid concentration (Figure 1a). Similarly, a twisted shaped graph is obtained in the case of $S_{\text {mix }}$ and solid lipid, as there was a non-linear increase in particle size with increase in $S_{\text {mix. }}$. Firstly, there was a decreasing trend in particle size up to the mid-level of $S_{\text {mix }}$, followed by a slight increase in the particle size. Whereas, in the case of solid lipid there was a sharp initial decline in the particle size followed by a rising trend (Figure 1b). Furthermore, (Figure 1c) depicted that with increase in $S_{\text {mix }}$ and liquid lipid concentration, the particle size tended to decrease first, followed by a non-linear increase in both the cases. With the increase in lipid concentration, an increase in particle size can be due to decrease in emulsifying efficiency of surfactant and thus, increase in particle agglomeration. Figure 1(d-f) depicts the 2Dcontour plots and 3D-response surface plots for \%EE. However, all the input variables had a positive influence on the $\% \mathrm{EE}$ of the drug, yet $\mathrm{S}_{\text {mix }}$ had a more pronounced effect. Both liquid lipid and solid lipid at all the levels positively influenced the \%EE of the drug. With increase in the liquid lipids concentration, there was an upsurge in the \%EE of drug up to a certain level and then it became constant. While in the case of solid lipid a non-linear increase in the \%EE was observed (figure 1d). However, it can be seen from the figure 1(e,f) that with increase in the $S_{\text {mix }}$, there was sharp rise in the \%EE of the drug vis-à-vis solid lipid and liquid lipid. This is attributed to the fact that high lipid and $S_{\text {mix }}$ concentration allows the better stabilization and solubilization of drug in the lipid blend, thus led to the enhancement of \%EE.

Optimized TQ-PNC formulation showed smallest particle size $(83.5 \mathrm{~nm})$ which was possibly due to the presence of optimal combination of PL90G and T80 (1:1) as $\mathrm{S}_{\text {mix }}$ at appropriate concentrations. The presence of nonionic surfactants and their blends with phospholipids often led to the particle stabilization.

PDI is a parameter, which is used to define the size distribution of the lipidic nanoconstructs. The PDI value (0.55) was in the acceptable range of uniformity. The acceptable value for PDI ranges between 0.05 to 0.7 , whereas the values greater than 0.7 indicate that the sample has a very broad size distribution $[41,42]$.The zeta potential value was close to zero $(-0.64)$, which is due to the presence of a non-ionic surfactant T80, responsible to stabilize systems by creating spatial inclusion $[43,44]$. The $\% \mathrm{EE}$ of the optimized PNC was found to be $94 \%$ revealing that the 
microemulsification method with the apt optimization of solid lipid, liquid lipid and $S_{\text {mix }}$ (T80:PL90G) can yield such high value of \%EE. Further, it has been observed that high phospholipid content reduces the risk of drug loss to the external phase, and thus provide more space to incorporate the drug, resulting in improved entrapment efficiency. This may also be due to an excess of phospholipid possibly forming multilayers around the particle.

The DSC thermogram of PNC does not show any peak pertaining to thymoquinone, which reveals the complete entrapment of TQ in the lipidic nanocarriers. The presence of both solid and liquid lipid mixture can lead to less ordered lipid crystalline arrangement. The shifting of TQ melting peak in the curves can be associated to the amorphous structure of PNC and complete solubilization of drug within the PNC [45]. The surface morphology of optimized PNCs reveal that the particles were spherical in shape, in agreement with the size i.e. below $100 \mathrm{~nm}$. SEM observation showed a dense solid spherical topography having smooth surface of the PNC. Moreover, no crystalline drug particles were visible. The XRD pattern of the pure TQ demonstrates high intensely peak, showing its crystalline nature compared to TQ-PNCs. A marked decrease in the intensity of TQ peak in TQ-PNC, indicating the solubility and stability of the drug in the PNC core. In FT-IR study, all the peaks relating to functional groups (hydroxyl, methyl, carbonyl) of all the excipients are intense and clear in the FTIR spectrum of the physical mixture. Thus, it can be inferred that there was no interaction between the drugs and the excipients, employed in various nanocarrier formulations. Also, the drug was stable and compatible with the excipients.

TQ-PNC showed significantly high drug release compared to pure TQ. It was observed that approximately $30 \%$ of the drug was released from PNC in first 2 hours vis-à-vis TQ followed by sustained release at a constant rate. This might be due to the presence of lipidic core and can be explained in three aspects, i.e., the presence of drug at the surface of PNCs, secondly liquid lipid and thirdly, smaller particle size [46]. In first case, TQ might be adsorbed at the surface of the nanoconstructs due to the presence of $S_{\text {mix }}$, secondly liquid lipid was distributed in solid lipid core, which leads to the formation of imperfect crystalline structure of PNC and thus increasing the rate of drug release [47]. In third case, smaller particle size in case of optimized PNC formulation offers the greater surface area, and hence, improves the contact between particles and dissolution medium. The best-fit model for PNCs was found to be Higuchi model indicating the controlled release mechanism of drug release from the nanoconstructs. This could be ascribed to the lipophilic 
nature of TQ, which is entrapped deeply in the lipidic core matrix in the solubilized form and thereby, slow diffusion of drug molecules via the phospholipid matrix of PNCs. The release profile of TQ from plain TQ suspension and optimized PNC formulation from GI fluids is shown in Figure 2(h). The initial burst of TQ release from the PNC may be due to the presence of TQ molecules on the surface closest to PNC particle. The sustained release phase may be associated to the diffusion of TQ molecules from the lipid core, which leads to the immobilization of drug from the PNC molecule. In addition, it may be because of the effect of decreasing TQ amount [48].

A stability test in simulated GI tract was designed to investigate the effect of composition on stability of nanocarriers. As said earlier the main aim behind stability study in simulated GI fluids was to first protect the drug from degradation from the harsh GI tract environment until it reaches the intestine. After reaching in the intestine, drug will be digested by intestinal enzyme to form mixed micelles composed of lipid degradation products (fatty acids, monoglycerides etc.), bile salts and phospholipids entrapping drug thus enhancing its bioavailability. The results showed the insignificant changes in all the formulation parameters viz. particle size, PDI and \%EE (Table 5). Although, PNC was found to be more stable in SIF compared to SGF. This stability of PNC in acidic $\mathrm{pH}$ might be due to presence of nonionic surfactants i.e., T80, which leads to the steric stabilization effect. These nonionic surfactants are unresponsive to flocculation and coalescence in low $\mathrm{pH}$ due to their molecular structure. Whereas in case of SIF particle aggregation can occur sometimes due to the release of surfactant layer from the surface of lipidic nanocarriers by lipase, thus leading to nanocarrier aggregation and exposing the surface of nanocarriers for lipid hydrolysis by intestinal enzymes [49].

The cellular uptake of PNC were assessed in Caco-2 cells [(Figure 2(i) (I-II) and 2(j)]. The particle size of PNC was found to play important role in the efficient uptake through GIT. The size of PNC was found to be less than $100 \mathrm{~nm}$. This confirmed the effective intestinal uptake, mostly in the lymphoid region, which helped in bypassing the first pass metabolism. In addition, the large surface area by nanosized PNC enhances the contact between the drug and the cell membrane, thereby augmenting the cell uptake of drug. Furthermore, the combined mechanism between lipids and surfactants can improve drug absorption by enhancing affinity for cell membranes with lipophilicity. Therefore, these results supported that TQ could be taken up into Caco-2 cells by amalgamation into PNC [50]. 
In in vivo animal pharmacokinetics, TQ-PNC showed 3.9-fold enhancement in the relative bioavailability vis-à-vis TQ-suspension, indicating the improved bioavailability of the drug in the lipid-based nanoparticles formulation. The improved pharmacokinetic and oral bioavailability of the TQ from lipidic matrix was probably because of combined promotion mechanisms. Firstly, the ability to adhere to gut wall. The ultrafine dispersions within nanometer range imparts them with a tremendous specific surface area and thus the possession of adhesion to gut wall, which is helpful to oral absorption. Secondly, the nano-sized formulation and the presence of $S_{\operatorname{mix}}$ as suitable solubilizers of TQ in lipidic core. Furthermore, faster absorption of TQ incorporated into lipidic matrix helped in avoiding first pass extensive gut wall metabolism due to close association of drug with lipid vis-a-vis plain TQ. Thirdly being a lipid-based system, PNCs may promote the uptake by M-cells in the Payer's patches and increase the absorption through the lymphatic pathway [51].

The observations from biochemical parameters showed that compared to the control group and all the groups including TQ-PNC showed significant decrease in ALP, ALT, AST, bilirubin and albumin level production ( $\mathrm{p}<0.001$ ) after PCM administration. Whereas reduction in the biomarker enzyme was more pronounced in the TQ-PNC group compared to all other groups ( $\mathrm{p}$ $<0.001)$. The effect may be assigned to the higher cellular permeability of TQ achieved by PNCs. PNCs with the blend of T80 and PL90G as a surfactant and co-surfactant, respectively, are anticipated to interact with the membrane lipids of liver cells resulting in physiologically significant effects as shown in this study [52]. Moreover, other hypothesis can be ascribed that the passage across endothelial fenestrations of carriers smaller than $100 \mathrm{~nm}$ can effectively target hepatic stellate cells HSCs (in rats). In addition, the presence of lipidic carrier facilitates capture by the liver and other organs. Overall, these results specified that optimized TQ-PNC showed enhanced hepatoprotective effects against PCM-induced liver damage. The results of biochemical examinations were further supported by the histopathological investigations in rats liver samples.

\section{Conclusion}

The present research work, have successfully prepared TQ-loaded PNCs and systemically optimized employing BBD, which helped in providing the optimal composition of three independent variables to select the best-optimized formulation. The prepared nanoconstructs demonstrated initial burst release followed by controlled release. Pharmacokinetic studies proved the potential of nanolipidic carriers to alleviate the challenge of lower bioavailability of TQ. 
Pharmacological effects of selected optimized PNC formulations showed significant reduction of various liver biomarkers compared to toxic group, which was further substantiated by histopathological examination of liver sections. Hence, we expect that these investigations can provide a new strategy for the development of nano-medicine and suggest TQ as a strong candidate for a new generation of hepato-protective molecule with reduced side effects in the not so distant future. The outcomes of the studies can be extrapolated to variety of bioactives and drugs with similar challenges and hurdles.

\section{Acknowledgement}

The authors are thankful to M/s Phospholipid GmbH, Nattermannallee, Germany, for the ex gratis supply of PL90G, and M/s Gattefosse, Saint-Priest, France, for providing the gift samples of Labrafac, Compritol 888 ATO and Labrafil. The authors are also thankful to Shoolini University for facilitating the research work.

\section{Declaration of interest}

The authors report no declarations of interest.

\section{Author contributions}

C Rathore carried out the study, helped in compilation of the data, manuscript writing and editing. $\mathrm{N}$ Upadhyay helped in conducting experimental work and interpreting analytical data. R Kaundal helped in editing and analysis of the data. RP Dwivedi helped in editing the data. S Rahatekar and A John helped in revising the manuscript. K Dua helped in interpretation of the data and checked the first draft of the prepared manuscript. M Tambuwala helped in revising the manuscript. S Jain and D Chaudhari helped in interpreting data and revising the manuscript. P Negi conceptualized, planned, supervised the study, analyzed, interpreted the data and edited the manuscript. All authors have read and approved the manuscript for publication 


\section{References}

1. Ahmed MF, Rao AS, Thayyil H, et al. Role of Melia azedarach leaf extract in Paracetamol Induced Hepatic damage in rats. Pharmacognosy Journal. 2011;1;3(21):60-4.

2. Singh A, Ahmad I, Akhter S, et al. Nanocarrier based formulation of Thymoquinone improves oral delivery: stability assessment, in vitro and in vivo studies. Colloids and Surfaces B: Biointerfaces. 2013;1(102):822-32.

3. Asrani SK, Devarbhavi H, Eaton J, et al. Burden of liver diseases in the world. Journal of hepatology. 2018.

4. Darakhshan $\mathrm{S}$, Pour $\mathrm{AB}$, Colagar $\mathrm{AH}$, et al. Thymoquinone and its therapeutic potentials. Pharmacological research. 2015;1(95):138-58.

5. Negi P, Rathore C, Sharma G, et al. Thymoquinone a Potential Therapeutic Molecule from the Plant Nigella sativa: Role of Colloidal Carriers in its Effective Delivery. Recent patents on drug delivery \& formulation. 2018;12(1):3-22.

6. Ballout F, Habli Z, Rahal ON, et al. Thymoquinone-based nanotechnology for cancer therapy: Promises and challenges. Drug discovery today. 2018;23(5):1089-98.

7. Kalam MA, Raish M, Ahmed A, et al. Oral bioavailability enhancement and hepatoprotective effects of thymoquinone by self-nanoemulsifying drug delivery system. Materials Science and Engineering: C. 2017;1(76):319-29.

8. Farkhondeh T, Samarghandian S, Shahri AM, et al. The neuroprotective effects of thymoquinone: A review. Dose-Response. 2018;16(2):1559325818761455

9. Shahid F, Farooqui Z, Khan AA, et al. Oral Nigella sativa oil and thymoquinone administration ameliorates the effect of long-term cisplatin treatment on the enzymes of carbohydrate metabolism, brush border membrane, and antioxidant defense in rat intestine. Naunyn-Schmiedeberg's archives of pharmacology. 2018;391(2):145-57.

10. Alkharfy KM, Ahmad A, Raish M, et al. Thymoquinone modulates nitric oxide production and improves organ dysfunction of sepsis. Life sciences. 2015;5(143):131-8.

11. Patra JK, Das G, Fraceto LF, et al., Nano based drug delivery system:recent development and future prospect. Journal of Nanobiotechnology. 2018;71:2-33

12. Sayeed S, Imam SS, Najmi AK, et al. Nonionic surfactant based thymoquinone loaded nanoproniosomal formulation: in vitro physicochemical evaluation and in vivo hepatoprotective efficacy. Drug development and industrial pharmacy. 2017;43(9):141320.

13. Salvi VR, Pawar P. Nanostructured lipid carriers (NLC) system: A novel drug targeting carrier. Journal of Drug Delivery Science and Technology. 2019;22:255-67

14. Pardeike J, Schwabe K, Müller RH. Influence of nanostructured lipid carriers (NLC) on the physical properties of the Cutanova Nanorepair Q10 cream and the in vivo skin hydration effect. International journal of pharmaceutics. 2010;396(1-2):166-73.

15. Raza K, Singh B, Singla N, et al. Nano-lipoidal carriers of isotretinoin with anti-aging potential: formulation, characterization and biochemical evaluation. Journal of drug targeting. 2013;21(5):435-42.

16. Porter CJ, Charman WN. Intestinal lymphatic drug transport: an update. Advanced drug delivery reviews. 2001;50(1-2):61-80.

17. Raza K, Singh B, Lohan S, et al. Nano-lipoidal carriers of tretinoin with enhanced percutaneous absorption, photostability, biocompatibility and anti-psoriatic activity. International journal of pharmaceutics. 2013;456(1):65-72. 
18. Abdelwahab SI, Sheikh BY, Taha MM, et al. Thymoquinone-loaded nanostructured lipid carriers: preparation, gastroprotection, in vitro toxicity, and pharmacokinetic properties after extravascular adginistration. International journal of nanomedicine. 2013;8:2163.

19. Ng WK, Saiful Yazan L, Yap LH, et al. Thymoquinone-loaded nanostructured lipid carrier exhibited cytotoxicity towards breast cancer cell lines (MDA-MB-231 and MCF-7) and cervical cancer cell lines (HeLa and SiHa). BioMed research international. 2015.

20. Khurana RK, Bansal AK, Beg S, et al. Enhancing biopharmaceutical attributes of phospholipid complex-loaded nanostructured lipidic carriers of mangiferin: systematic development, characterization and evaluation. International journal of pharmaceutics. 2017;518(1-2):289-306.

21. Singh B, Kapil R, Nandi M, et al. Developing oral drug delivery systems using formulation by design: vital precepts, retrospect and prospects. Expert opinion on drug delivery. 2011;8(10):1341-60.

22. Ferreira SC, Bruns RE, Ferreira HS, et al. Box-Behnken design: an alternative for the optimization of analytical methods. Analytica chimica acta. 2007;10;(2):179-86.

23. Rakić T, Kasagić-Vujanović I, Jovanović M, et al. Comparison of full factorial design, central composite design, and box-behnken design in chromatographic method development for the determination of fluconazole and its impurities. Analytical Letters. 2014;47(8):1334-47.

24. Negi P, Singh B, Sharma G, et al. Biocompatible lidocaine and prilocaine loadednanoemulsion system for enhanced percutaneous absorption: QbD-based optimisation, dermatokinetics and in vivo evaluation. Journal of microencapsulation. 2015;32(5):41931.

25. Souto EB, Wissing SA, Barbosa CM, et al. Development of a controlled release formulation based on SLN and NLC for topical clotrimazole delivery. International Journal of Pharmaceutics. 2004;278(1):71-7.

26. Rathore C, Upadhyay NK, Sharma A, et al. Phospholipid nanoformulation of thymoquinone with enhanced bioavailability: Development, characterization and antiinflammatory activity. Journal of Drug Delivery Science and Technology. 2019;23.

27. Raza K, Singh B, Singal P, et al. Systematically optimized biocompatible isotretinoinloaded solid lipid nanoparticles (SLNs) for topical treatment of acne. Colloids and Surfaces B: Biointerfaces. 2013;105:67-74.

28. Negi P, Singh B, Sharma G, et al. Phospholipid microemulsion-based hydrogel for enhanced topical delivery of lidocaine and prilocaine: QbD-based development and evaluation. Drug Deliv. (2014) doi: 10.3109/10717544.2014.923067.

29. Negi P, Aggarwal M, Sharma G, et al. Niosome-based hydrogel of resveratrol for topical applications: An effective therapy for pain related disorder(s). Biomedicine \& Pharmacotherapy. 2017;88:480-487.

30. Szüts A, Budai-Szücs M, Erös I, et al. Study of gel-forming properties of sucrose esters for thermosensitive drug delivery systems. International journal of pharmaceutics. 2010;383(1-2):132-7.

31. Chadha R, Saini A, Arora P, et al. Multicomponent solids of lamotrigine with some selected coformers and their characterization by thermoanalytical, spectroscopic and X-ray diffraction methods. CrystEngComm. 2011;13(20):6271-84. 
32. Jain AK, Thanki K, Jain S. Solidified self-nanoemulsifying formulation for oral delivery of combinatorial therapeutic regimen: part I. Formulation development, statistical optimization, and in vitro characterization. Pharmaceutical research. 2014;1(31-4):923-45.

33. Rathore C, Jain N, Garg N, et al. Polysaccharide-microsponge based matrix tablet for colon targeting of ketoprofen: in vitro and in vivo evidence. International journal of pharmaceutical sciences and research. 2017;1;8(10):4250-60.

34. Bapat P, Ghadi R, Chaudhari D, Katiyar SS, Jain S. Tocophersolan stabilized lipid nanocapsules with high drug loading to improve the permeability and oral bioavailability of curcumin. International journal of pharmaceutics. 2019 Apr 5;560:219-27.

35. Pathan SA, Jain GK, Zaidi SM, et al. Stability-indicating ultra-performance liquid chromatography method for the estimation of thymoquinone and its application in biopharmaceutical studies. Biomedical chromatography. 2011;25(5):613-20.

36. Das S, Roy P, Auddy RG, et al. Silymarin nanoparticle prevents paracetamol-induced hepatotoxicity. International journal of nanomedicine. 2011;6:1291.

37. Baravalia Y, Vaghasiya Y, Chanda S. Hepatoprotective effect of Woodfordia fruticosa Kurz flowers on diclofenac sodium induced liver toxicity in rats. Asian Pacific Journal of Tropical Medicine. 2011;4(5):342-6.

38. Danaei M, Dehghankhold M, Ataei S, et al. Impact of Particle Size and Polydispersity Index on the Clinical Applications of Lipidic Nanocarrier Systems. Pharmaceutics. 2018;10:1-17

39. Shah M, Agrawal Y. Ciprofloxacin hydrochloride-loaded glyceryl monostearate nanoparticle: factorial design of Lutrol F68 and Phospholipon 90G. Journal of microencapsulation. 2012;29(4):331-43.

40. Bunjes H, Koch MH, Westesen K. Effects of surfactants on the crystallization and polymorphism of lipid nanoparticles. In Molecular Organisation on Interfaces 2002;7-10.

41. Hasan AA, Sabry SA, Abdallah MH, et al. Formulation and in vitro characterization of poly (DL-lactide-co-glycolide)/eudragit RLPO or RS30D nanoparticles as an oral carrier of levofloxacin hemihydrate. Pharmaceutical development and technology. 2016;21(6):655-63.

42. Danaei M, Dehghankhold M, Ataei S, et al. Impact of particle size and polydispersity index on the clinical applications of lipidic nanocarrier systems. Pharmaceutics. 2018;10(2):57.

43. Kovačević AB, Müller RH, Savić SD, et al. Solid lipid nanoparticles (SLN) stabilized with polyhydroxy surfactants: preparation, characterization and physical stability investigation. Colloids and Surfaces A: Physicochemical and Engineering Aspects. 2014;444:15-25.

44. Zirak MB, Pezeshki A. Effect of surfactant concentration on the particle size, stability and potential zeta of beta carotene nano lipid carrier. Int J Curr Microbiol Appl Sci. 2015;4(9):924-32.

45. Baloch J, Sohail MF, Sarwar HS, Kiani MH, Khan GM, Jahan S, et al. SelfNanoemulsifying Drug Delivery System (SNEDDS) for Improved Oral Bioavailability of Chlorpromazine: In Vitro and In Vivo Evaluation. Medicina (B Aires). Multidisciplinary Digital Publishing Institute; 2019; 55:210. 
46. Bashiri S, Ghanbarzadeh B, Ayaseh A, et al. Preparation and characterization of chitosancoated nanostructured lipid carriers (CH-NLC) containing cinnamon essential oil for enriching milk and anti-oxidant activity. LWT. 2019; 13:108836.

47. Carbinatto FM, de Castro AD, Evangelista RC, et al. Insights into the swelling process and drug release mechanisms from cross-linked pectin/high amylose starch matrices. Asian journal of pharmaceutical sciences. 2014;9(1):27-34.

48. Uprit S, Sahu RK, Roy A, et al. Preparation and characterization of minoxidil loaded nanostructured lipid carrier gel for effective treatment of alopecia. Saudi Pharmaceutical Journal. 2013;21(4):379-385.

49. Wang K, Qi J, Weng T, et al. Enhancement of oral bioavailability of cyclosporine A: comparison of various nanoscale drug-delivery systems. International journal of nanomedicine. 2014;9:4991.

50. Singh N, Khullar N, Kakkar V, et al. Hepatoprotective effects of sesamol loaded solid lipid nanoparticles in carbon tetrachloride induced sub-chronic hepatotoxicity in rats. Environmental toxicology. 2016;31(5):520-32.

51. Aditya NP, Shim M, Lee I et al. Curcumin and Genistein Co-loaded Nanostructured Lipid Carriers: in vitro Digestion and Antiprostate Cancer Activity. Journal of food agricultural and food chemistry.2013;61:1878-83.

52. Son GH, Na YG, Huh HW, et al. Systemic Design and Evaluation of Ticagrelor-Loaded Nanostructured Lipid Carriers for Enhancing Bioavailability and Antiplatelet Activity. Phjarmaceutics.2019;11:1-18.

53. Algul D, Duman G, Ozdemir S, Preformulation, Characterization, and In Vitro Release studies of Caffeine-Loaded Solid Lipid Nanoparticles. Journal of Cosmetic Science.2018;69:165-173. 
Figure 1. Two-dimensional contour plots and corresponding three-dimensional response surface plot depicting the effect of various input variables on; (a) Particle size; Liquid lipid $\left(\mathrm{X}_{2}\right)$, solid lipid (X1); (b) $\mathrm{S}_{\text {mix }}\left(\mathrm{X}_{3}\right)$, solid lipid $\left(\mathrm{X}_{1}\right)$; (c) $\mathrm{S}_{\text {mix }}\left(\mathrm{X}_{3}\right)$, Liquid lipid $\left(\mathrm{X}_{2}\right)$ and effect of various input variables on \%EE (d) Liquid lipid $\left(\mathrm{X}_{2}\right)$, solid lipid (X1); (e) $\mathrm{S}_{\text {mix }}\left(\mathrm{X}_{3}\right)$, solid lipid $\left(\mathrm{X}_{1}\right)$; (f) $\mathrm{S}_{\text {mix }}\left(\mathrm{X}_{3}\right)$, Liquid lipid $\left(\mathrm{X}_{2}\right)$.

Figure 2. (a) Particle size distribution of optimized PNC formulation, (b) DSC graphs of drug, PNC, capmul and stearic acid, (c) TEM microphotograph (at 60,000X) of TQ-loaded PNCs (d) FE-SEM microphotograph (at 30,000X) of TQ-loaded PNCs, (e) XRD spectra of pure drug, PNC, sucrose, capmul and stearic acid .(f) Overlay FTIR spectrum of drug and the excipients A: TQ, B: Physical mixture, C: Sucrose, D: Stearic acid, (g) Comparative in vitro release of optimized TQPNC and pure TQ, (h) Comparative in vitro release of optimized TQ-PNC and pure TQ in SGF and SIF, (i) Qualitative cell uptake of C-6 loaded PNC (I) Free C-6 and (II) C-6 loaded PNC, where (a) images under green fluorescence channel of C-6 pannel (b) corresponding differential interface contrast image of the cell (c) superimposition of the panel (a and b), Panel (d) and (e) in all the images shows vertical and horizontal line series of fluorescence along with the yellow line of the image (c) respectively. (j) The time dependent cell uptake of pure TQ and PNC by Caco-2 cells

Figure 3(A). Plasma concentration-time profile curve of TQ after oral administration of optimized PNC and TQ suspension (mean $\pm \mathrm{SD}, \mathrm{n}=6$ ) (B) Effect of various formulations on serum and tissue biochemical parameters. Data is presented as Mean $\pm \operatorname{SD}(n=4)$ and analyses by one-way analysis of variance followed by Tukey's Multiple Comparison Test. $\mathrm{a}^{P}<0.001$ vs saline control, $\mathrm{b}^{P}<$ 0.001 vs toxic group and $c^{P}<0.05$ vs standard. (C) a) Normal control showed normal sinusoidal architecture and no fibrosis; b) Toxic group showed Parenchymal cell injury with lymphocytes and macrophages infiltration, along with inflammation and apoptosis; c) TQ suspension group showed mild lymphocytic portal infiltrate; d) TQ-PNC showed few lymphocytes and normal vacuoles are present in the cells and e) Silymarin group showed normal hepatocytes cell and few scattered foci 
a)
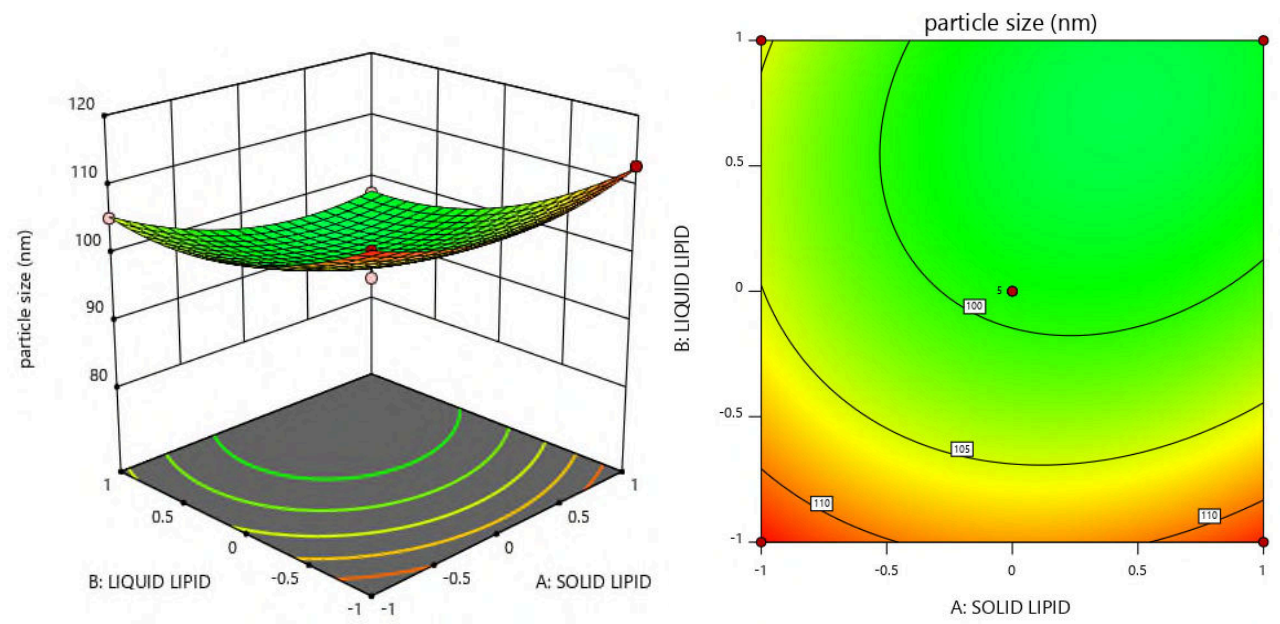

b)
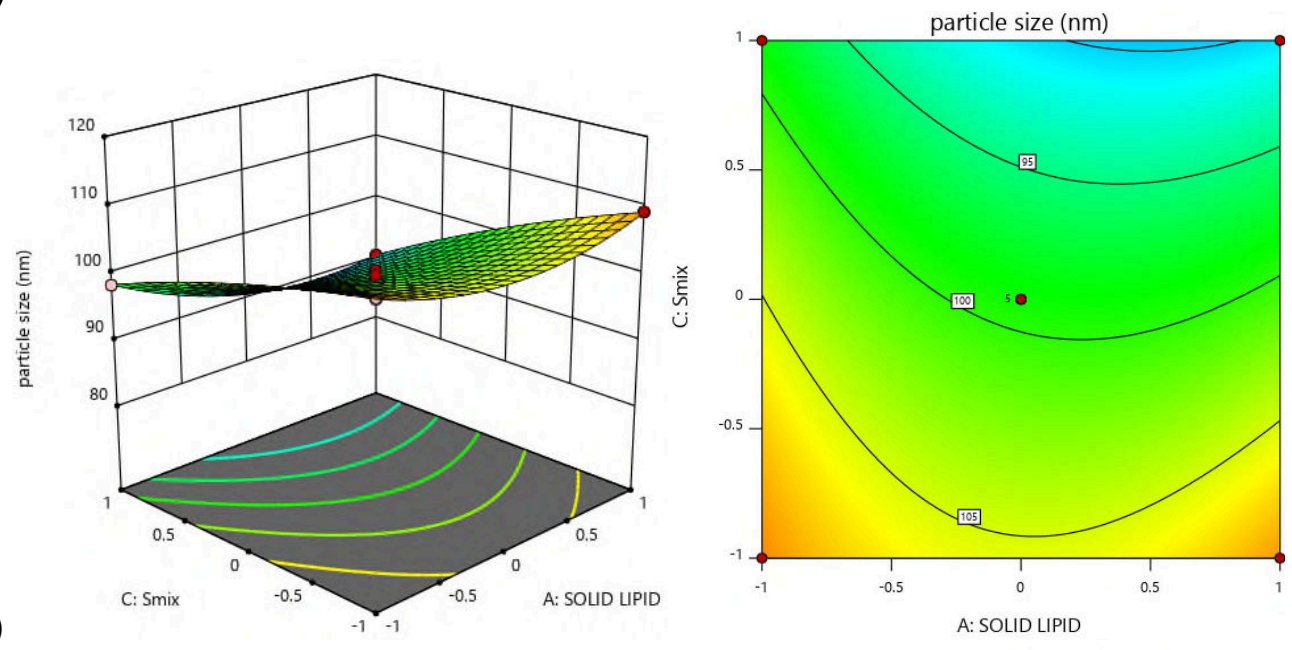

c)
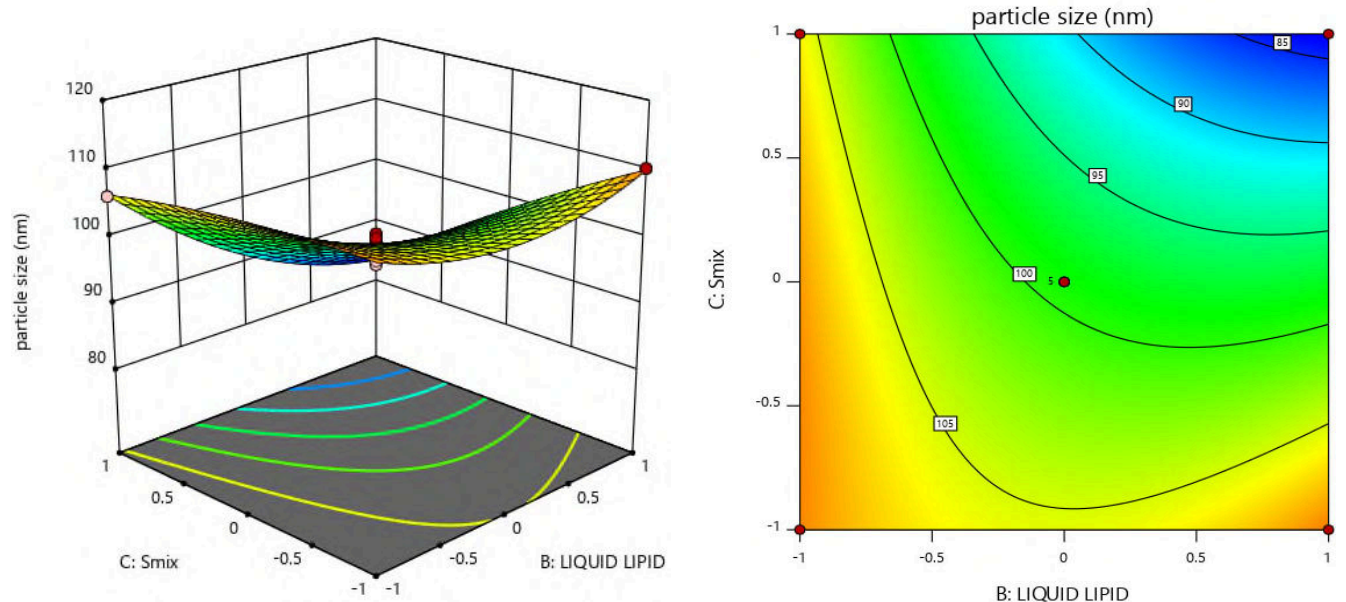
d)

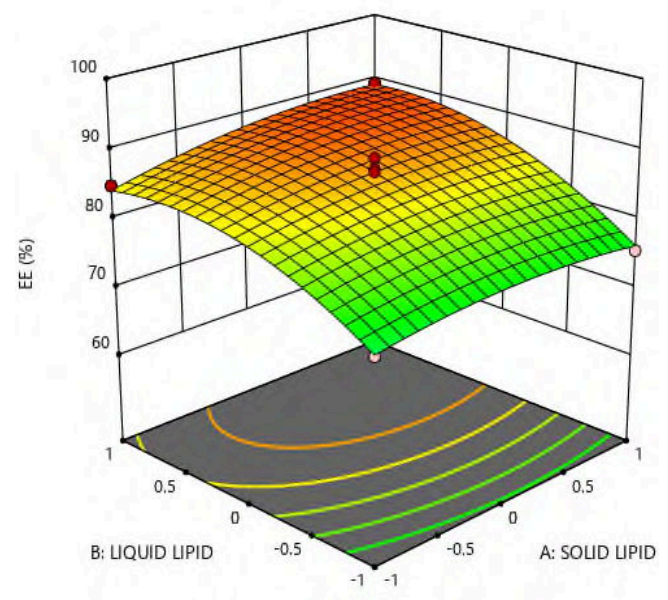

e)

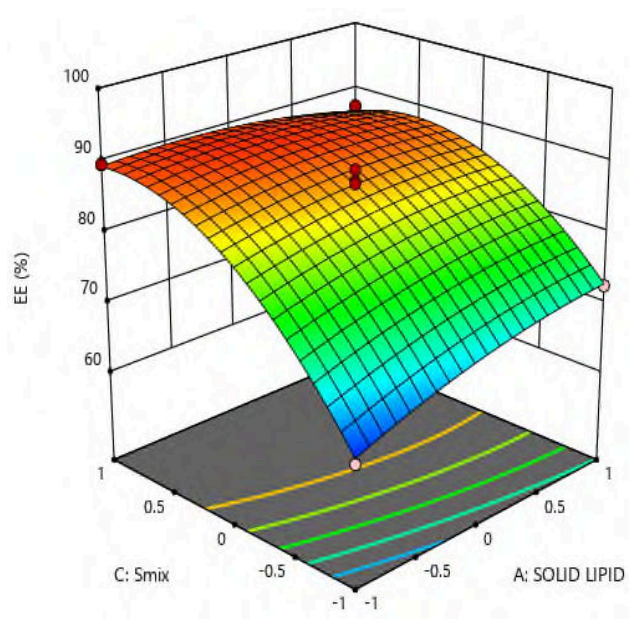

f)

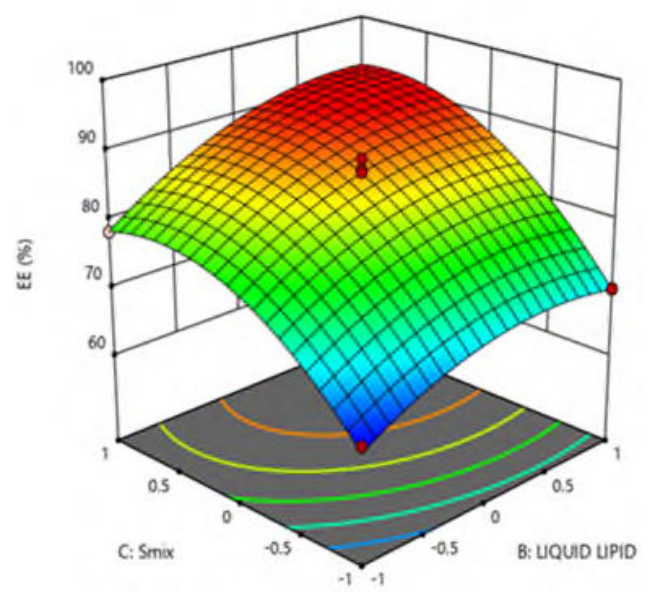

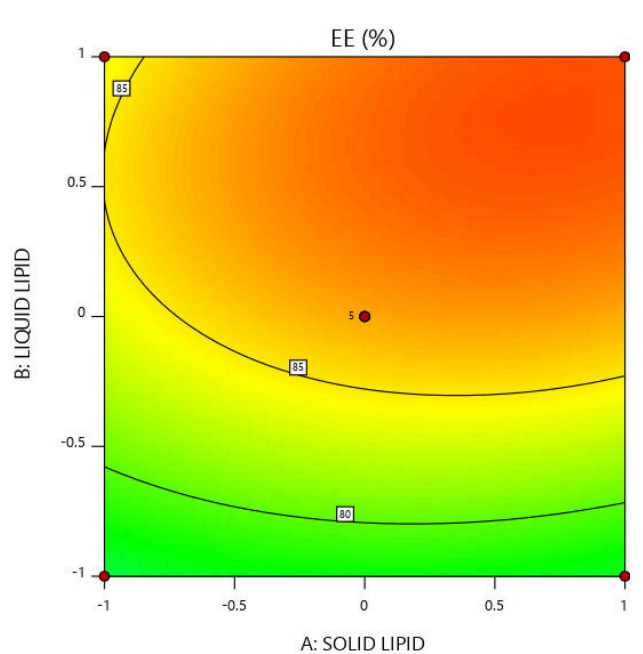
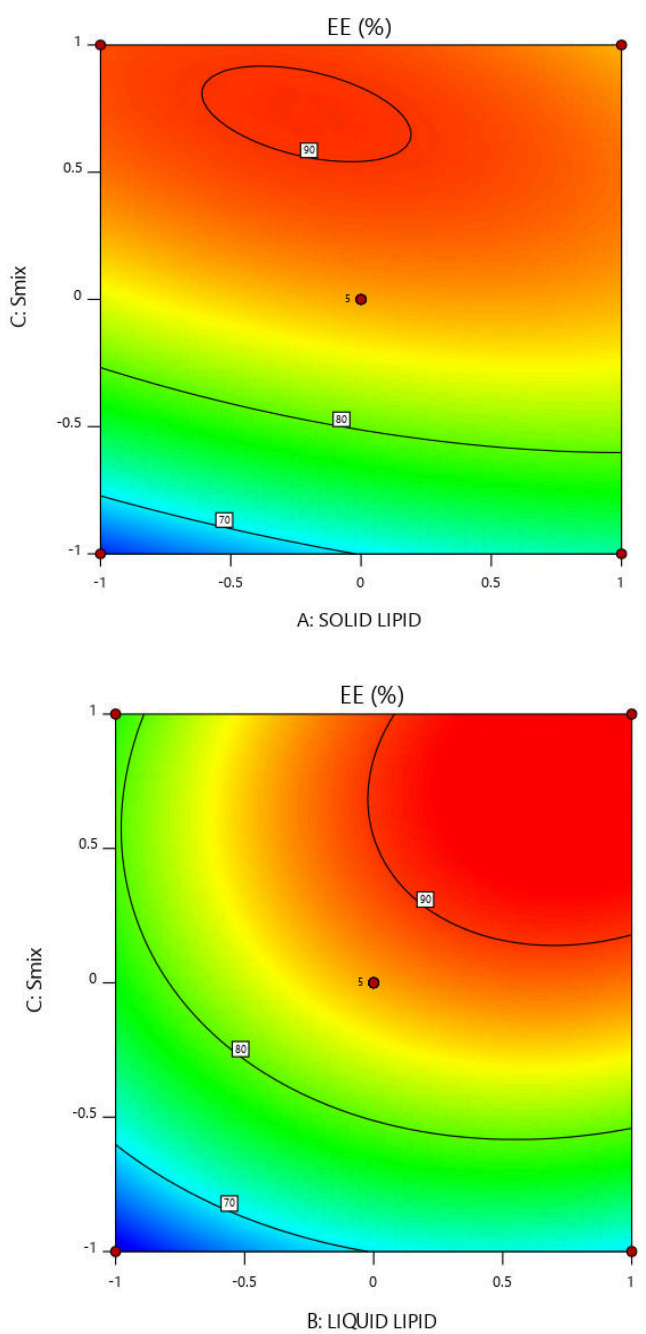

Figure 1 

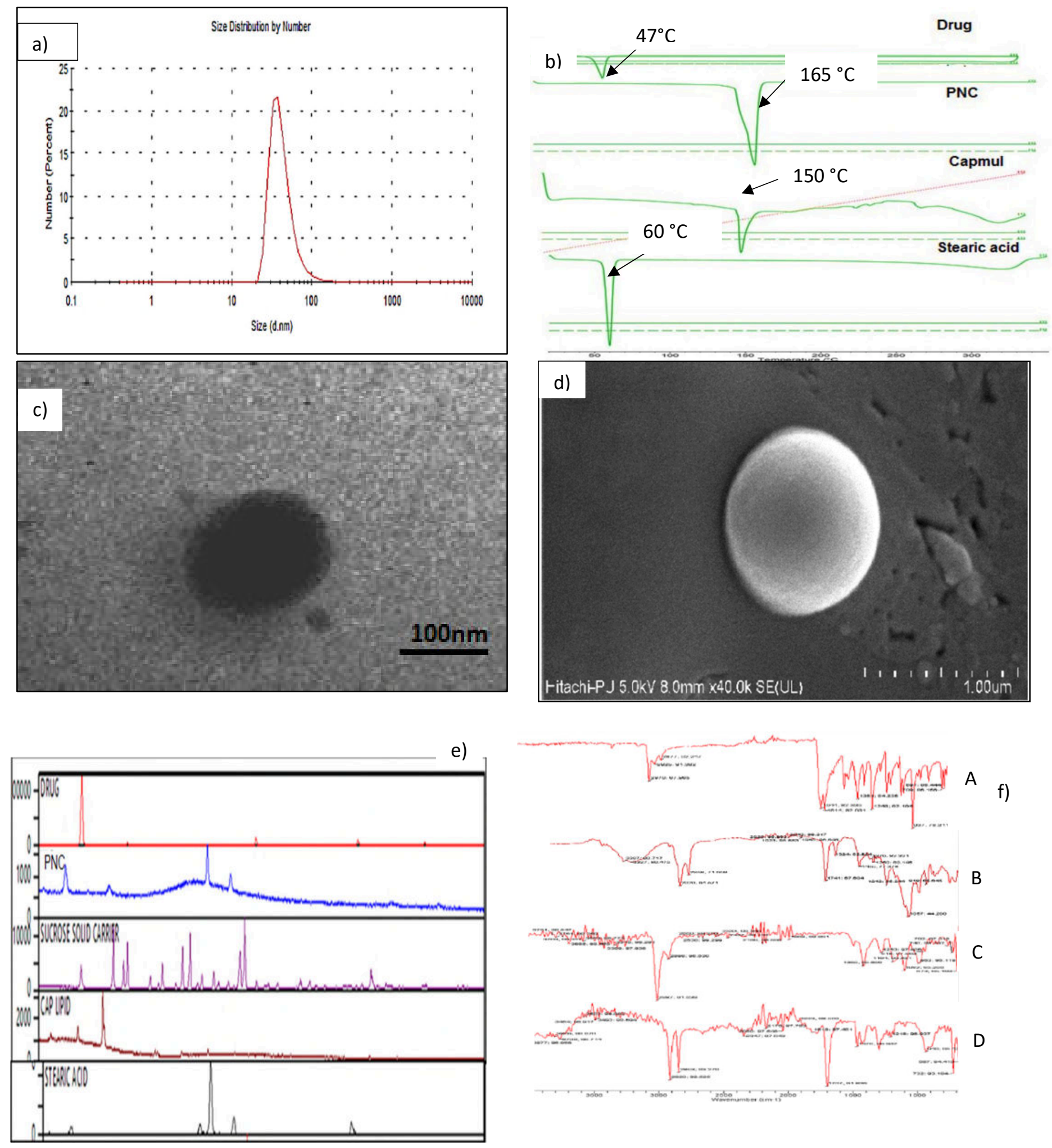

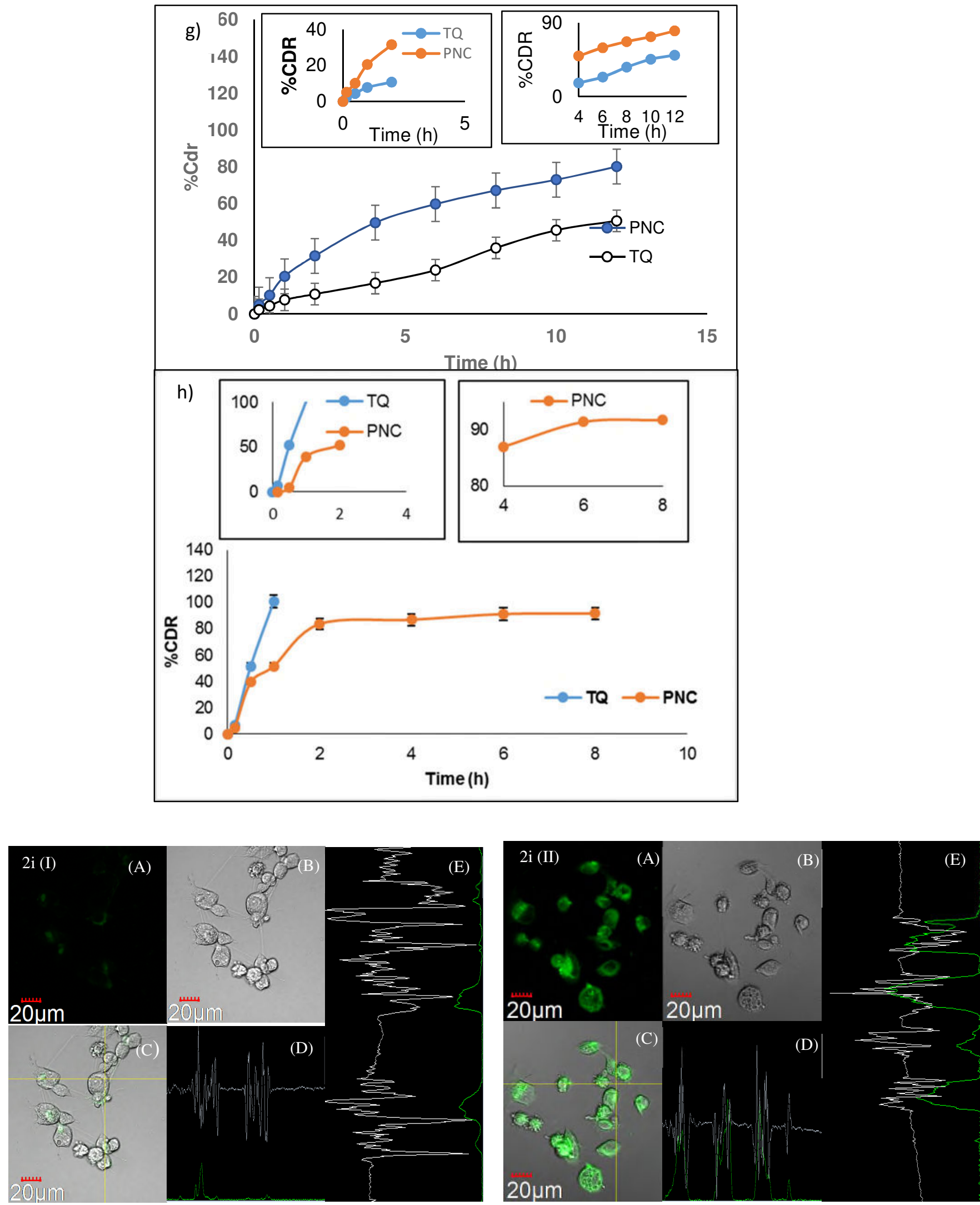


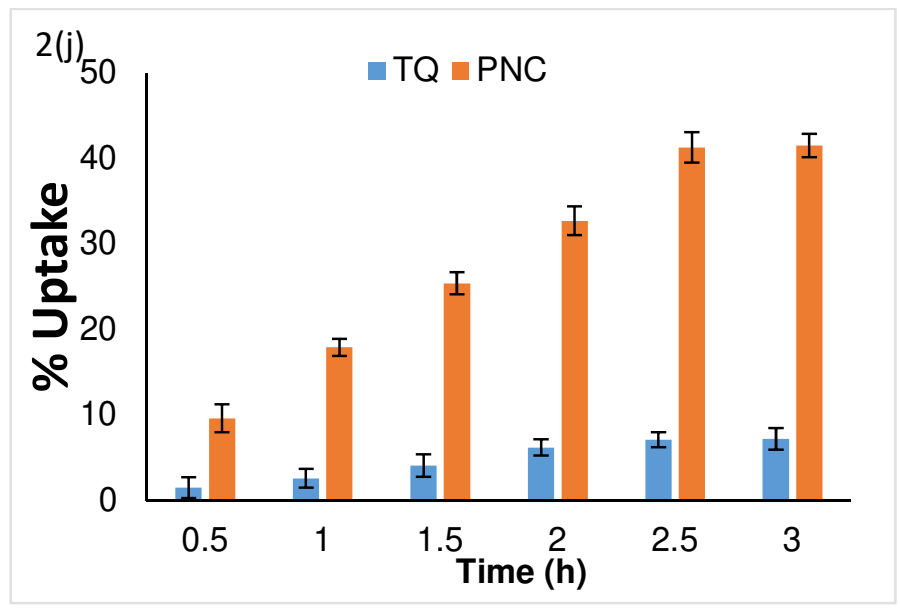

Figure 2 (a-j)

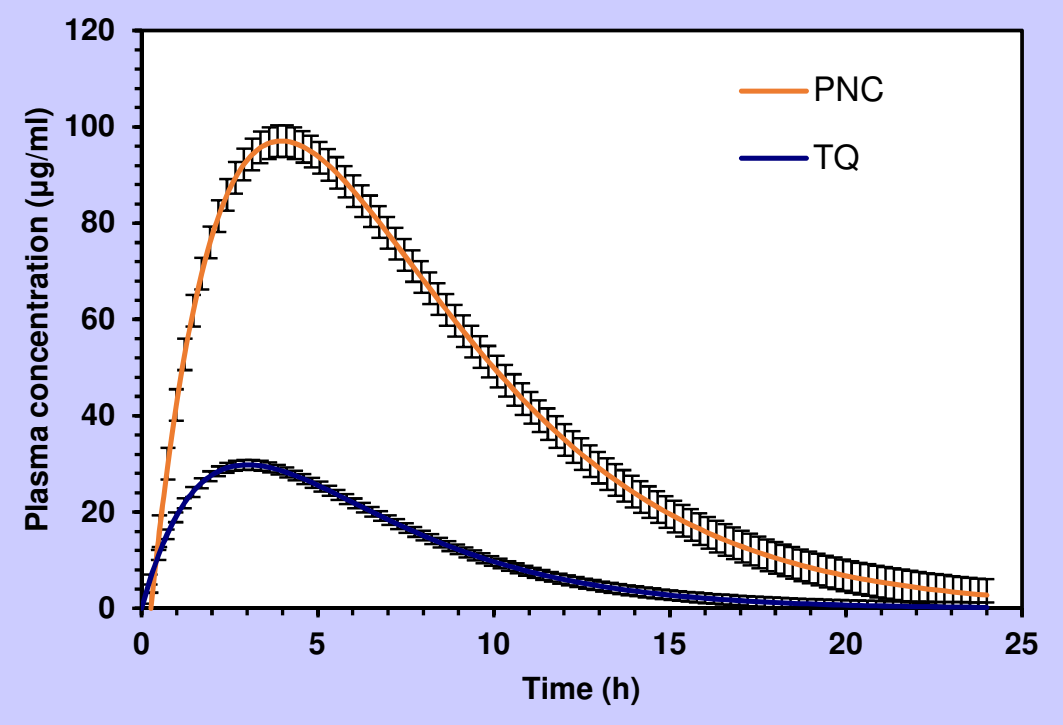

Figure 3 (A) 


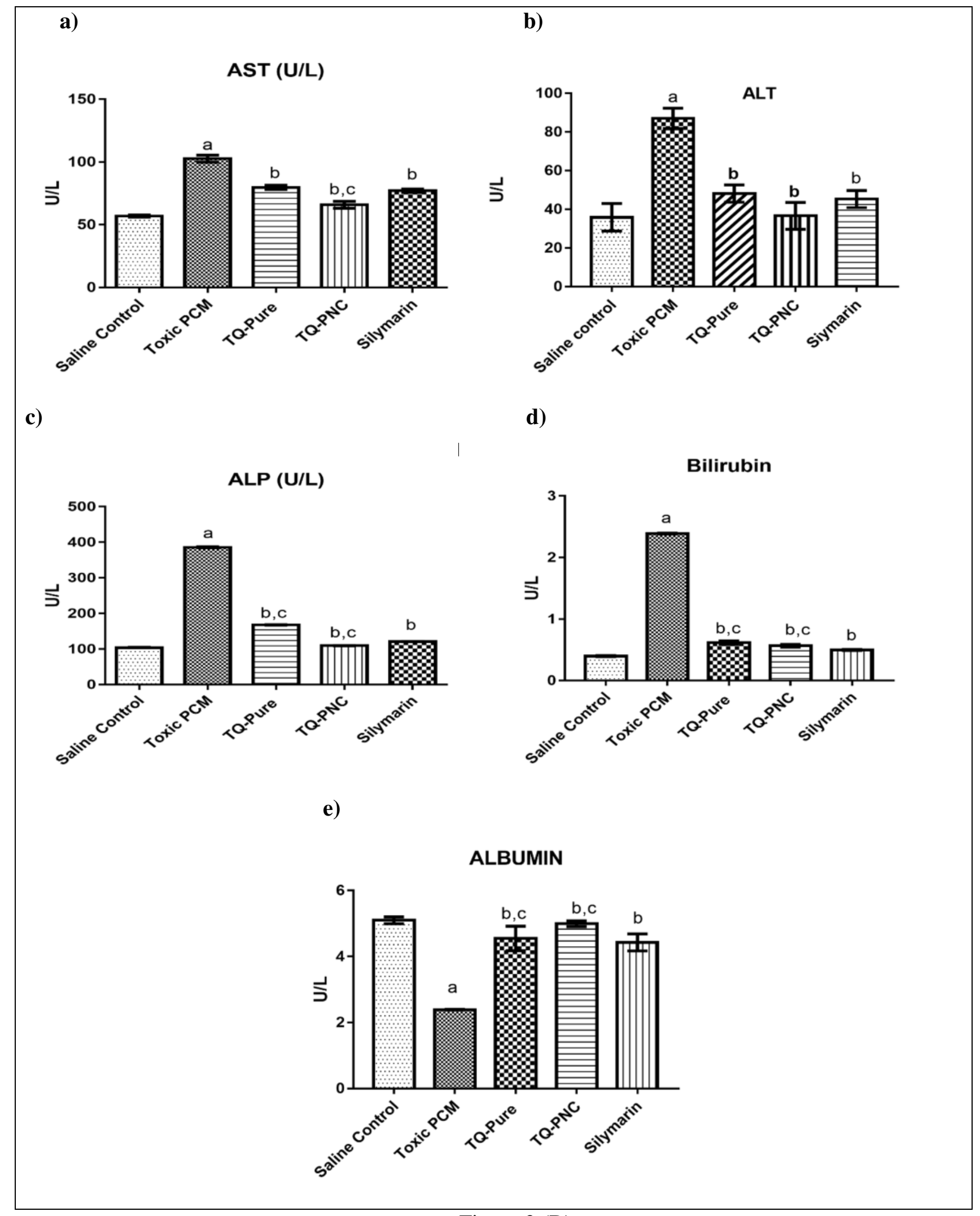

Figure 3 (B) 

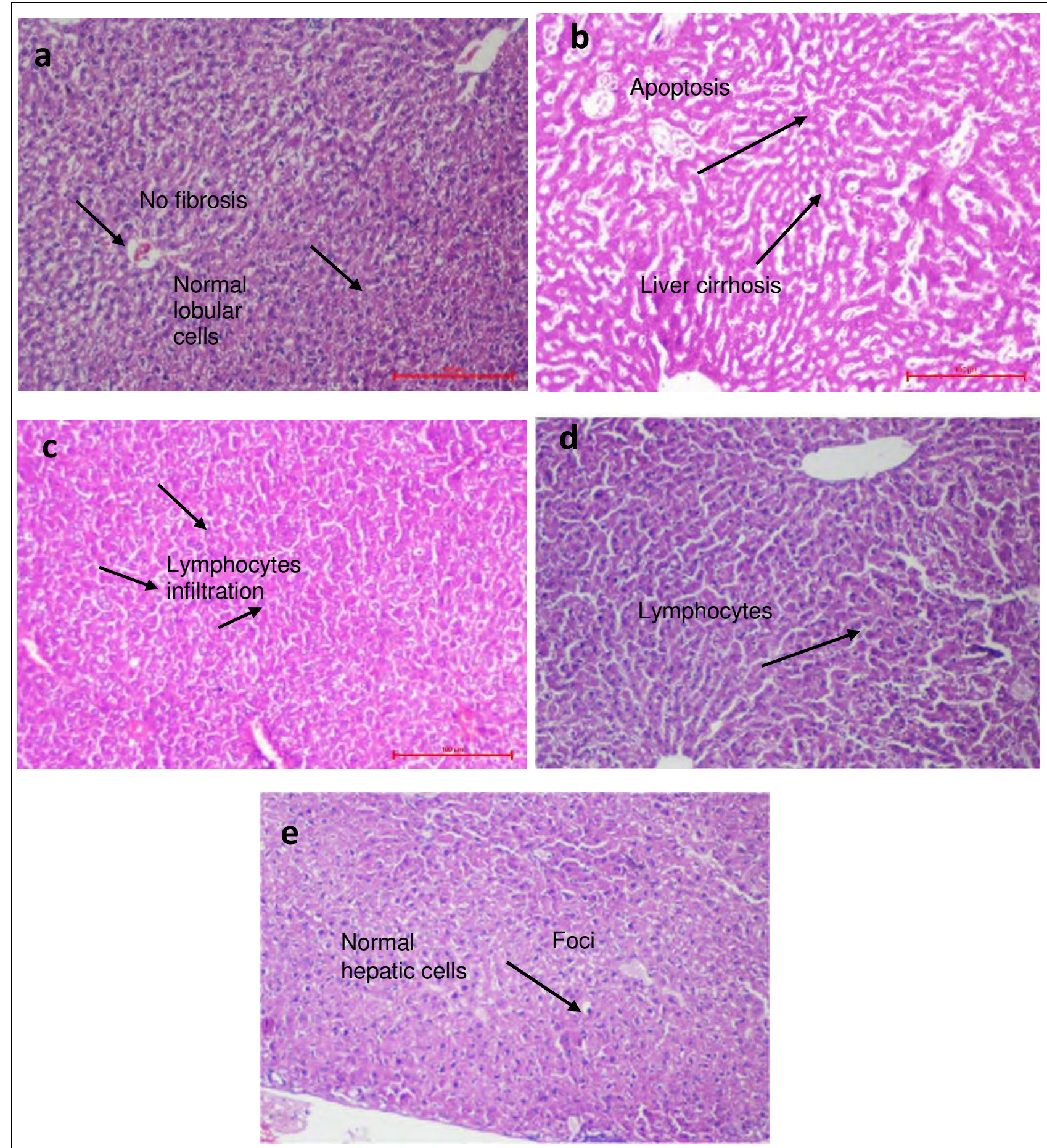

Figure 3 (C) 
Table 1

Experimental runs of BBD design matrix and their responses

\section{Table 2}

Constraints for numeric optimization and predicted solutions

Table 3

Comparison of experimental results with predicted responses

\section{Table 4}

Drug-excipient interaction employing FT-IR

\section{Table 5}

Stability of PNC in simulated GI fluids

\section{Table 6}

Pharmacokinetic parameters (mean \pm SD) after oral administration of TQ -PNC and TQ suspension in rats $(n=6)$

\section{Table 7}

Effects of optimized TQ-PNC and TQ treatments on Serum and Tissue Biochemical Parameters $($ mean $\pm \mathrm{SD}, \mathrm{n}=4)$ 
Table 1

\begin{tabular}{|c|c|c|c|c|c|}
\hline Runs & Solid Lipid & Liquid lipid & $\begin{array}{l}{ }^{*} S_{\operatorname{mix}} \\
(T 80: P L 90 G)\end{array}$ & $\% E E$ & Particle size $(\mathrm{nm})$ \\
\hline 1 & 0 & 0 & 0 & 99.2 & 88.9 \\
\hline 2 & 1 & 1 & 0 & 97.5 & 89.3 \\
\hline 3 & 0 & 1 & 1 & 83.78 & 91.5 \\
\hline 4 & -1 & 0 & 1 & 98.4 & 89.6 \\
\hline 5 & 0 & 0 & 0 & 99.2 & 87.3 \\
\hline 6 & 1 & -1 & 0 & 112.8 & 75.5 \\
\hline 7 & 0 & 1 & -1 & 110.2 & 69.9 \\
\hline 8 & 0 & 0 & 0 & 100.6 & 85.5 \\
\hline 9 & 1 & 0 & -1 & 109.2 & 72.5 \\
\hline 10 & 0 & -1 & 1 & 106.1 & 78.3 \\
\hline 11 & -1 & 0 & -1 & 109.6 & 63.5 \\
\hline 12 & 0 & -1 & -1 & 109.3 & 63.3 \\
\hline 13 & -1 & -1 & 0 & 113.6 & 75.3 \\
\hline 14 & 1 & 0 & 1 & 90.5 & 87.3 \\
\hline 15 & 0 & 0 & 0 & 99.9 & 86.9 \\
\hline 16 & 0 & 0 & 0 & 96.5 & 85.5 \\
\hline 17 & -1 & 1 & 0 & 105.3 & 84.9 \\
\hline \multirow{2}{*}{\multicolumn{3}{|c|}{ Independent variables (g) }} & \multicolumn{3}{|c|}{ Level used, actual (coded) } \\
\hline & & & $\operatorname{Low}(-1)$ & Medium (0) & $\operatorname{High}(+1)$ \\
\hline \multicolumn{3}{|c|}{ Solid Lipid } & -1 & 0 & +1 \\
\hline \multicolumn{3}{|c|}{ Liquid Lipid } & -1 & 0 & +1 \\
\hline \multicolumn{3}{|l|}{$\mathrm{S}_{\text {mix }}$} & -1 & 0 & +1 \\
\hline
\end{tabular}

${ }^{*}$ T80:PL90G was fixed in the ratio of 1:1 
Table 2

\begin{tabular}{|c|c|c|c|c|c|c|}
\hline Variable & Goal & $\begin{array}{l}\text { Lower } \\
\text { limit }\end{array}$ & \multicolumn{2}{|c|}{ Upper limit } & \multicolumn{2}{|c|}{ Importance } \\
\hline A:Solid lipid & maximize & -1 & \multicolumn{2}{|c|}{1} & \multicolumn{2}{|c|}{ 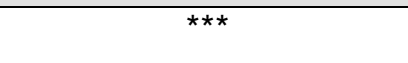 } \\
\hline B:Liquid lipid & is in range & -1 & \multicolumn{2}{|c|}{1} & \multicolumn{2}{|c|}{$* * *$} \\
\hline$C: S_{\text {mix }}$ & maximize & -1 & \multicolumn{2}{|c|}{1} & \multicolumn{2}{|c|}{ 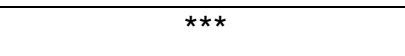 } \\
\hline Particle size & minimize & 83.78 & \multicolumn{2}{|c|}{113.6} & \multicolumn{2}{|c|}{$* * *$} \\
\hline \%EE & is in range & 63.3 & \multicolumn{2}{|c|}{91.5} & \multicolumn{2}{|c|}{$\star \star \star$} \\
\hline A & B & C & $\begin{array}{l}\text { Particle } \\
\text { size }\end{array}$ & \%EE & Desirability & \\
\hline 1 & 0.69 & 1 & 83.44 & 90.17 & 1.000 & Selected \\
\hline 1 & 0.85 & 1 & 82.37 & 90.48 & 1.000 & \\
\hline 1 & 0.83 & 1 & 82.52 & 90.45 & 1.000 & \\
\hline 1 & 0.938 & 1 & 81.93 & 90.55 & 1.000 & \\
\hline 1 & 0.668 & 1 & 83.61 & 90.11 & 1.000 & \\
\hline 1 & 0.993 & 1 & 81.66 & 90.57 & 1.000 & \\
\hline 1 & 0.972 & 1 & 81.79 & 90.57 & 1.000 & \\
\hline
\end{tabular}


Table 3

\begin{tabular}{|c|c|c|c|c|}
\hline Composition & $\begin{array}{c}\text { Response } \\
\text { Variable }\end{array}$ & $\begin{array}{c}\text { Predicted } \\
\text { Value }\end{array}$ & $\begin{array}{c}\text { Experimental } \\
\text { Value }\end{array}$ & $\begin{array}{c}\text { \% Prediction } \\
\text { Error }\end{array}$ \\
\hline \multirow{3}{*}{ OPT } & Particle size $(\mathrm{nm})$ & 83.44 & 83.47 & -0.04 \\
\cline { 2 - 5 } & \%EE & 90.18 & 90.12 & 0.07 \\
\hline \multirow{2}{*}{ VAL-1 } & Particle size $(\mathrm{nm})$ & 82.37 & 82.39 & -0.02 \\
\cline { 2 - 5 } & \%EE & 90.48 & 90.39 & 0.10 \\
\hline \multirow{2}{*}{ VAL-2 } & Particle size $(\mathrm{nm})$ & 82.52 & 82.42 & 0.10 \\
\cline { 2 - 5 } & \%EE & 90.45 & 90.51 & -0.07 \\
\hline \multirow{2}{*}{ VAL-3 } & Particle size $(\mathrm{nm})$ & 82.06 & 82.12 & -0.10 \\
\cline { 2 - 5 } & \%EE & 91.00 & 90.90 & 0.11 \\
\hline \multirow{2}{*}{ VAL-4 } & Particle size $(\mathrm{nm})$ & 82.76 & 82.70 & 0.06 \\
\cline { 2 - 5 } & \%EE & 91.00 & 90.81 & 0.21 \\
\hline \multirow{2}{*}{ VAL-5 } & Particle size $(\mathrm{nm})$ & 81.93 & 82.00 & -0.07 \\
\cline { 2 - 5 } & \%EE & 90.54 & 90.50 & 0.04 \\
\hline \multirow{2}{*}{ VAL-6 } & Particle size $(\mathrm{nm})$ & 83.61 & 83.5 & 0.11 \\
\cline { 2 - 5 } & \%EE & 90.19 & 90.10 & 0.10 \\
\hline
\end{tabular}

Table 4

\begin{tabular}{|c|c|c|}
\hline Reported Peaks $\left(\mathrm{cm}^{-1}\right)$ & Observed Peaks $\left(\mathrm{cm}^{-1}\right)$ & Assignment \\
\hline \multicolumn{3}{|c|}{ TQ } \\
\hline $1670-1820$ & 1644,1614 & $-\mathrm{C}=\mathrm{O}$ stretch \\
\hline $2850-3000$ & $2970,2925,2877$ & $-\mathrm{CH}$ stretch \\
\hline $1350-1480$ & 1360 & - $\mathrm{CH}$ bending \\
\hline \multicolumn{3}{|c|}{ Physical Mixture } \\
\hline $2850-3000$ & 2929,2853 & -CH stretch \\
\hline $1670-1820$ & 1741 & $\mathrm{C}=\mathrm{O}$ stretch \\
\hline $1900-2000$ & 1920,1950 & $\mathrm{C}=\mathrm{C}$ stretch \\
\hline $3200-3600$ & 3327,3387 & $-\mathrm{OH}$ stretch \\
\hline $1350-1480$ & 1350,1376 & -CH stretch \\
\hline \multicolumn{3}{|c|}{ Sucrose } \\
\hline $2850-3000$ & 2987 & -CH stretch \\
\hline $3500-3700$ & 3665 & $-\mathrm{OH}$ stretch \\
\hline $1350-1480$ & 1395 & $-\mathrm{CH}$ stretch \\
\hline \multicolumn{3}{|c|}{ Stearic acid } \\
\hline $3200-3600$ & 3493 & $-\mathrm{OH}$ stretch \\
\hline $2850-3000$ & 2920 & -CH stretch \\
\hline $1670-1820$ & 1707 & $-\mathrm{C}=\mathrm{O}$ stretch \\
\hline
\end{tabular}


Table 5

\begin{tabular}{|c|c|c|c|c|c|c|}
\hline \multirow[t]{2}{*}{ Fluids } & \multicolumn{2}{|c|}{ Particle size (nm) } & \multicolumn{2}{|c|}{ PDI } & \multicolumn{2}{|c|}{$\% \mathbf{E E}$} \\
\hline & Initial & Final & Initial & Final & Initial & Final \\
\hline $\begin{array}{c}\text { SGF } \\
(\mathrm{pH} 1.2)\end{array}$ & \multirow{2}{*}{83.5} & 81.30 & \multirow{2}{*}{0.55} & 0.52 & \multirow{2}{*}{93.4} & 92.5 \\
\hline $\begin{array}{c}\text { SIF } \\
(\mathrm{pH} 6.8)\end{array}$ & & 89.4 .5 & & 0.59 & & 93.5 \\
\hline
\end{tabular}

Table 6

\begin{tabular}{|l|l|l|}
\hline Parameters & TQ suspension & TQ-PNC \\
\hline $\mathrm{C}_{\max }(\mu \mathrm{g} / \mathrm{mL})$ & 29.83 & 97.06 \\
\hline $\mathrm{t}_{1 / 2}(\mathrm{~h})$ & 2.13 & 2.61 \\
\hline $\mathrm{t}_{\max }(\mathrm{h})$ & 3.01 & 3.96 \\
\hline $\mathrm{V}_{\mathrm{d}}(\mathrm{L} / \mathrm{kg})$ & 0.25 & 0.07 \\
\hline $\mathrm{CL}(\mathrm{L} / \mathrm{kg} / \mathrm{h})$ & 0.08 & 0.02 \\
\hline $\mathrm{Ke}$ & 0.32 & 0.26 \\
\hline AUC $(0-24)(\mu \mathrm{g} \cdot \mathrm{h} / \mathrm{mL})$ & 243.53 & 965.14 \\
\hline AUC(0-inf) $(\mu \mathrm{gg} \cdot \mathrm{h} / \mathrm{mL})$ & 244.29 & 976.29 \\
\hline $\begin{array}{l}\text { Relative bioavailability (F) } \\
(\text { Ratio of AUC }(0-\mathrm{t}) \text { of TQ-PNC } \\
\text { to TQ suspension) }(\mathrm{F})(\%)\end{array}$ & & 399.64 \\
\hline
\end{tabular}

Table 7

\begin{tabular}{|l|l|l|l|l|l|}
\hline \multicolumn{7}{|c|}{$($ Mean \pm SD) $(\mathbf{n}=4)$} \\
\hline Groups & ALT (U/L) & ALP (U/L) & AST (U/L) & $\begin{array}{l}\text { Bilirubin } \\
(\mathbf{m g} / \mathbf{d l})\end{array}$ & Albumin \\
\hline Saline control & $35.9 \pm 10.46$ & $104.41 \pm 2.54$ & $59.2 \pm 3.21$ & $0.40 \pm 0.01$ & $5.1 \pm 0.06$ \\
\hline Toxic control (PCM) & $87.0 \pm 6.49$ & $386.1 \pm 5.54$ & $102.73 \pm 5.65$ & $2.39 \pm 0.01$ & $3.2 \pm 0.08$ \\
\hline TQ suspension & $47.41 \pm 7.64$ & $168.20 \pm 2.01$ & $79.86 \pm 4.65$ & $0.60 \pm 0.02$ & $4.8 \pm 0.21$ \\
\hline TQ-PNC & $39.2 \pm 9.23$ & $110 \pm 1.67$ & $69.7 \pm 2.55$ & $0.58 \pm 0.03$ & $4.5 \pm 0.04$ \\
\hline Silymarin & $45.3 \pm 6.01$ & $121.31 \pm 1.62$ & $73.90 \pm 3.54$ & $0.50 \pm 0.01$ & $4.3 \pm 0.10$ \\
\hline
\end{tabular}

Values were the means of three replicate samples. The data were presented as mean \pm SD $(n=4),{ }^{a} p<0.001$ vs saline control, ${ }^{b} \mathrm{p}<0.001$ vs toxic control and ${ }^{c} \mathrm{p}<0.05$ vs Silymarin 


\title{
References
}

\section{Papers of special note have been highlighted as:}

\author{
* of interest \\ ** of considerable interest
}

1. Ahmed MF, Rao AS, Thayyil H, et al. Role of Melia azedarach leaf extract in Paracetamol Induced Hepatic damage in rats. Pharmacognosy Journal. 2011;1;3(21):60-4.

2. Singh A, Ahmad I, Akhter S, et al. Nanocarrier based formulation of Thymoquinone improves oral delivery: stability assessment, in vitro and in vivo studies. Colloids and Surfaces B: Biointerfaces. 2013;1(102):822-32.

\section{**This research article reports the use of nanocarrier for liver cirrhosis and explain the absorption mechanism followed by nanocarriers}

3. Asrani SK, Devarbhavi H, Eaton J, et al. Burden of liver diseases in the world. Journal of hepatology. 2018.

4. Darakhshan $\mathrm{S}$, Pour $\mathrm{AB}$, Colagar $\mathrm{AH}$, et al. Thymoquinone and its therapeutic potentials. Pharmacological research. 2015;1(95):138-58.

\section{**Excellent review on pharmacological potential of TQ and its mechanism of action in various diseases}

5. Negi P, Rathore C, Sharma G, et al. Thymoquinone a Potential Therapeutic Molecule from the Plant Nigella sativa: Role of Colloidal Carriers in its Effective Delivery. Recent patents on drug delivery \& formulation. 2018;12(1):3-22.

6. Ballout F, Habli Z, Rahal ON, et al. Thymoquinone-based nanotechnology for cancer therapy: Promises and challenges. Drug discovery today. 2018;23(5):1089-98.

7. Kalam MA, Raish M, Ahmed A, et al. Oral bioavailability enhancement and hepatoprotective effects of thymoquinone by self-nanoemulsifying drug delivery system. Materials Science and Engineering: C. 2017;1(76):319-29.

8. Farkhondeh T, Samarghandian S, Shahri AM, et al. The neuroprotective effects of thymoquinone: A review. Dose-Response. 2018;16(2):1559325818761455

9. Shahid F, Farooqui Z, Khan AA, et al. Oral Nigella sativa oil and thymoquinone administration ameliorates the effect of long-term cisplatin treatment on the enzymes of carbohydrate metabolism, brush border membrane, and antioxidant defense in rat intestine. Naunyn-Schmiedeberg's archives of pharmacology. 2018;391(2):145-57.

10. Alkharfy KM, Ahmad A, Raish M, et al. Thymoquinone modulates nitric oxide production and improves organ dysfunction of sepsis. Life sciences. 2015;5(143):131-8.

11. Patra JK, Das G, Fraceto LF, et al., Nano based drug delivery system:recent development and future prospect. Journal of Nanobiotechnology. 2018;71:2-33

12. Sayeed S, Imam SS, Najmi AK, et al. Nonionic surfactant based thymoquinone loaded nanoproniosomal formulation: in vitro physicochemical evaluation and in vivo 
hepatoprotective efficacy. Drug development and industrial pharmacy. 2017;43(9):141320.

13. Salvi VR, Pawar P. Nanostructured lipid carriers (NLC) system: A novel drug targeting carrier. Journal of Drug Delivery Science and Technology. 2019;22:255-67

14. Pardeike J, Schwabe K, Müller RH. Influence of nanostructured lipid carriers (NLC) on the physical properties of the Cutanova Nanorepair Q10 cream and the in vivo skin hydration effect. International journal of pharmaceutics. 2010;396(1-2):166-73.

15. Raza K, Singh B, Singla N, et al. Nano-lipoidal carriers of isotretinoin with anti-aging potential: formulation, characterization and biochemical evaluation. Journal of drug targeting. 2013;21(5):435-42.

16. Porter CJ, Charman WN. Intestinal lymphatic drug transport: an update. Advanced drug delivery reviews. 2001;50(1-2):61-80.

17. Raza K, Singh B, Lohan S, et al. Nano-lipoidal carriers of tretinoin with enhanced percutaneous absorption, photostability, biocompatibility and anti-psoriatic activity. International journal of pharmaceutics. 2013;456(1):65-72.

18. Abdelwahab SI, Sheikh BY, Taha MM, et al. Thymoquinone-loaded nanostructured lipid carriers: preparation, gastroprotection, in vitro toxicity, and pharmacokinetic properties after extravascular adginistration. International journal of nanomedicine. 2013;8:2163.

19. Ng WK, Saiful Yazan L, Yap LH, et al. Thymoquinone-loaded nanostructured lipid carrier exhibited cytotoxicity towards breast cancer cell lines (MDA-MB-231 and MCF-7) and cervical cancer cell lines (HeLa and SiHa). BioMed research international. 2015.

20. Khurana RK, Bansal AK, Beg S, et al. Enhancing biopharmaceutical attributes of phospholipid complex-loaded nanostructured lipidic carriers of mangiferin: systematic development, characterization and evaluation. International journal of pharmaceutics. 2017;518(1-2):289-306.

21. Singh B, Kapil R, Nandi M, et al. Developing oral drug delivery systems using formulation by design: vital precepts, retrospect and prospects. Expert opinion on drug delivery. 2011;8(10):1341-60.

22. Ferreira SC, Bruns RE, Ferreira HS, et al. Box-Behnken design: an alternative for the optimization of analytical methods. Analytica chimica acta. 2007;10;(2):179-86.

23. Rakić T, Kasagić-Vujanović I, Jovanović M, et al. Comparison of full factorial design, central composite design, and box-behnken design in chromatographic method development for the determination of fluconazole and its impurities. Analytical Letters. 2014;47(8):1334-47.

24. Negi P, Singh B, Sharma G, et al. Biocompatible lidocaine and prilocaine loadednanoemulsion system for enhanced percutaneous absorption: QbD-based optimisation, dermatokinetics and in vivo evaluation. Journal of microencapsulation. 2015;32(5):41931.

\section{**An excellent paper describing the BBD optimization technique}

25. Souto EB, Wissing SA, Barbosa CM, et al. Development of a controlled release formulation based on SLN and NLC for topical clotrimazole delivery. International Journal of Pharmaceutics. 2004;278(1):71-7. 
26. Rathore C, Upadhyay NK, Sharma A, et al. Phospholipid nanoformulation of thymoquinone with enhanced bioavailability: Development, characterization and antiinflammatory activity. Journal of Drug Delivery Science and Technology. 2019;23.

27. Raza K, Singh B, Singal P, et al. Systematically optimized biocompatible isotretinoinloaded solid lipid nanoparticles (SLNs) for topical treatment of acne. Colloids and Surfaces B: Biointerfaces. 2013;105:67-74.

28. Negi P, Singh B, Sharma G, et al. Phospholipid microemulsion-based hydrogel for enhanced topical delivery of lidocaine and prilocaine: QbD-based development and evaluation. Drug Deliv. (2014) doi: 10.3109/10717544.2014.923067.

29. Negi P, Aggarwal M, Sharma G, et al. Niosome-based hydrogel of resveratrol for topical applications: An effective therapy for pain related disorder(s). Biomedicine \& Pharmacotherapy. 2017;88:480-487.

30. Szüts A, Budai-Szücs M, Erös I, et al. Study of gel-forming properties of sucrose esters for thermosensitive drug delivery systems. International journal of pharmaceutics. 2010;383(1-2):132-7.

31. Chadha R, Saini A, Arora P, et al. Multicomponent solids of lamotrigine with some selected coformers and their characterization by thermoanalytical, spectroscopic and X-ray diffraction methods. CrystEngComm. 2011;13(20):6271-84.

32. Jain AK, Thanki K, Jain S. Solidified self-nanoemulsifying formulation for oral delivery of combinatorial therapeutic regimen: part I. Formulation development, statistical optimization, and in vitro characterization. Pharmaceutical research. 2014;1(31-4):923-45.

33. Rathore C, Jain N, Garg N, et al. Polysaccharide-microsponge based matrix tablet for colon targeting of ketoprofen: in vitro and in vivo evidence. International journal of pharmaceutical sciences and research. 2017;1;8(10):4250-60.

34. Bapat P, Ghadi R, Chaudhari D, Katiyar SS, Jain S. Tocophersolan stabilized lipid nanocapsules with high drug loading to improve the permeability and oral bioavailability of curcumin. International journal of pharmaceutics. 2019 Apr 5;560:219-27.

\section{** An important report describing Caco-2 cells uptake study employing coumarin-6 dye}

35. Pathan SA, Jain GK, Zaidi SM, et al. Stability-indicating ultra-performance liquid chromatography method for the estimation of thymoquinone and its application in biopharmaceutical studies. Biomedical chromatography. 2011;25(5):613-20.

\section{* An important paper explaining the analytical method for quantification of TQ}

36. Das S, Roy P, Auddy RG, et al. Silymarin nanoparticle prevents paracetamol-induced hepatotoxicity. International journal of nanomedicine. 2011;6:1291.

37. Baravalia Y, Vaghasiya Y, Chanda S. Hepatoprotective effect of Woodfordia fruticosa Kurz flowers on diclofenac sodium induced liver toxicity in rats. Asian Pacific Journal of Tropical Medicine. 2011;4(5):342-6. 
38. Danaei M, Dehghankhold M, Ataei S, et al. Impact of Particle Size and Polydispersity Index on the Clinical Applications of Lipidic Nanocarrier Systems. Pharmaceutics. 2018;10:1-17

39. Shah M, Agrawal Y. Ciprofloxacin hydrochloride-loaded glyceryl monostearate nanoparticle: factorial design of Lutrol F68 and Phospholipon 90G. Journal of microencapsulation. 2012;29(4):331-43.

40. Bunjes H, Koch MH, Westesen K. Effects of surfactants on the crystallization and polymorphism of lipid nanoparticles. In Molecular Organisation on Interfaces 2002;7-10.

41. Hasan AA, Sabry SA, Abdallah MH, et al. Formulation and in vitro characterization of poly (DL-lactide-co-glycolide)/eudragit RLPO or RS30D nanoparticles as an oral carrier of levofloxacin hemihydrate. Pharmaceutical development and technology. 2016;21(6):655-63.

42. Danaei M, Dehghankhold M, Ataei S, et al. Impact of particle size and polydispersity index on the clinical applications of lipidic nanocarrier systems. Pharmaceutics. 2018;10(2):57.

43. Kovačević AB, Müller RH, Savić SD, et al. Solid lipid nanoparticles (SLN) stabilized with polyhydroxy surfactants: preparation, characterization and physical stability investigation. Colloids and Surfaces A: Physicochemical and Engineering Aspects. 2014;444:15-25.

44. Zirak MB, Pezeshki A. Effect of surfactant concentration on the particle size, stability and potential zeta of beta carotene nano lipid carrier. Int J Curr Microbiol Appl Sci. 2015;4(9):924-32.

45. Bashiri S, Ghanbarzadeh B, Ayaseh A, et al. Preparation and characterization of chitosancoated nanostructured lipid carriers (CH-NLC) containing cinnamon essential oil for enriching milk and anti-oxidant activity. LWT. 2019; 13:108836.

46. Carbinatto FM, de Castro AD, Evangelista RC, et al. Insights into the swelling process and drug release mechanisms from cross-linked pectin/high amylose starch matrices. Asian journal of pharmaceutical sciences. 2014;9(1):27-34.

47. Uprit S, Sahu RK, Roy A, et al. Preparation and characterization of minoxidil loaded nanostructured lipid carrier gel for effective treatment of alopecia. Saudi Pharmaceutical Journal. 2013;21(4):379-385.

48. Wang K, Qi J, Weng T, et al. Enhancement of oral bioavailability of cyclosporine A: comparison of various nanoscale drug-delivery systems. International journal of nanomedicine. 2014;9:4991.

\section{*A good paper explaining the various mechanisms of drug release from nanocarriers}

49. Singh N, Khullar N, Kakkar V, et al. Hepatoprotective effects of sesamol loaded solid lipid nanoparticles in carbon tetrachloride induced sub-chronic hepatotoxicity in rats. Environmental toxicology. 2016;31(5):520-32.

50. Aditya NP, Shim M, Lee I et al. Curcumin and Genistein Co-loaded Nanostructured Lipid Carriers: in vitro Digestion and Antiprostate Cancer Activity. Journal of food agricultural and food chemistry.2013;61:1878-83. 
51. Son GH, Na YG, Huh HW, et al. Systemic Design and Evaluation of Ticagrelor-Loaded Nanostructured Lipid Carriers for Enhancing Bioavailability and Antiplatelet Activity. Phjarmaceutics.2019;11:1-18.

52. Algul D, Duman G, Ozdemir S, Preformulation, Characterization, and In Vitro Release studies of Caffeine-Loaded Solid Lipid Nanoparticles. Journal of Cosmetic Science.2018;69:165-173. 


\section{Enhanced oral bioavailability and hepatoprotective activity of \\ thymoquinone in the form of phospholipidic nano-constructs}

Rathore, C.

Taylor and Francis

Rathore C, Upadhyay N, Kaundal R, et al., (2020) Enhanced oral bioavailability and hepatoprotective activity of thymoquinone in the form of phospholipidic nano-constructs. Expert Opinion on Drug Delivery, Volume 17, Issue 2, 2020, pp. 237-253

https://doi.org/10.1080/17425247.2020.1716728

Downloaded from Cranfield Library Services E-Repository 